\title{
Synectin promotes fibrogenesis by regulating PDGFR isoforms through distinct mechanisms
}

Mary C. Drinane, ${ }^{1}$ Usman Yaqoob, ${ }^{1}$ Haibin Yu, ${ }^{1,2}$ Fanghong Luo, ${ }^{1,3}$ Thomas Greuter, ${ }^{1}$ Juan P. Arab, ${ }^{1}$ Enis Kostallari, ${ }^{1}$ Vikas K. Verma, ${ }^{1}$ Jessica Maiers, ${ }^{1}$ Thiago Milech De Assuncao, ${ }^{1}$ Michael Simons, ${ }^{4}$ Debabrata Mukhopadhyay, ${ }^{5}$ Tatiana Kisseleva, ${ }^{6}$ David A. Brenner, ${ }^{6}$ Raul Urrutia, ${ }^{1}$ Cwen Lomberk, Yandong Gao,, Giovanni Ligresti, ${ }^{7}$ Daniel J. Tschumperlin, ${ }^{7}$ Alexander Revzin, ${ }^{7}$ Sheng Cao, ${ }^{1}$ and Vijay H. Shah ${ }^{1}$

'Gastroenterology Research Unit, Division of Gastroenterology and Hepatology, Mayo Clinic, Rochester, Minnesota, USA. ${ }^{2}$ Beijing Youan Hospital, Capital Medical University, Beijing, China. ${ }^{3}$ Medical College, Xiamen University, Xiamen, Fujian, China. ${ }^{4}$ Section of Cardiovascular Medicine, Yale University, New Haven, Connecticut, USA. ${ }^{5}$ Department of Biochemistry and Molecular Biology, Mayo Clinic, Jacksonville, Florida, USA. ${ }^{6}$ Department of Surgery, UCSD, California, USA.

'Department of Physiology and Biomedical Engineering, Mayo Clinic, Rochester, Minnesota, USA.

The scaffold protein synectin plays a critical role in the trafficking and regulation of membrane receptor pathways. As platelet-derived growth factor receptor (PDGFR) is essential for hepatic stellate cell (HSC) activation and liver fibrosis, we sought to determine the role of synectin on the PDGFR pathway and development of liver fibrosis. Mice with deletion of synectin from HSC were found to be protected from liver fibrosis. mRNA sequencing revealed that knockdown of synectin in HSC demonstrated reductions in the fibrosis pathway of genes, including PDGFR- $\beta$. Chromatin IP assay of the PDGFR- $\beta$ promoter upon synectin knockdown revealed a pattern of histone marks associated with decreased transcription, dependent on $\mathrm{p} 300$ histone acetyltransferase. Synectin knockdown was found to downregulate PDGFR- $\alpha$ protein levels, as well, but through an alternative mechanism: protection from autophagic degradation. Site-directed mutagenesis revealed that ubiquitination of specific PDGFR- $\alpha$ lysine residues was responsible for its autophagic degradation. Furthermore, functional studies showed decreased PDGF-dependent migration and proliferation of HSC after synectin knockdown. Finally, human cirrhotic livers demonstrated increased synectin protein levels. This work provides insight into differential transcriptional and posttranslational mechanisms of synectin regulation of PDGFRs, which are critical to fibrogenesis.

Authorship note: M.C. Drinane, U. Yaqoob, H. Yu, and F. Luo contributed equally to this work.

Conflict of interest: The authors have declared that no conflict of interest exists.

Submitted: January 13, 2017 Accepted: November 22, 2017 Published: December 21, 2017

Reference information: JCI Insight. 2017;2(24):e92821. https://doi.org/10.1172/jci. insight.92821.

\section{Introduction}

The discovery of the molecular basis underlying the pathophysiological mechanisms of human disease is a step of paramount importance for the discovery of effective therapies. In this regard, while advanced liver fibrosis accounts for significant morbidity and mortality through complications of portal hypertension, liver failure, and cancer, effective treatments remain to be developed. Our laboratory focuses on studying hepatic stellate cell (HSC), since they are key regulators of liver fibrosis (1-3). In response to various stimuli, HSC assume a myofibroblast phenotype characterized by increased migration, proliferation, and release of extracellular matrix proteins. HSC migration is conspicuous in fibrogenesis and also because their motility wraps adjacent endothelial cells and constrict sinusoids, thereby elevating intravascular pressure and leading to morbid portal hypertension (4). Thus, it follows that extending our understanding of how alterations in the biology of HSC occur during the fibrogenic processes may pave the way toward the development of effective treatments for many liver diseases.

HSC are enriched in both platelet-derived growth factor receptor- $\alpha$ (PDGFR- $\alpha$ ) and $-\beta$, which have been demonstrated to play critical roles in the development of cirrhosis (5-7). Notably, extensive studies have readily demonstrated that these pathways initiate and propagate HSC-mediated liver fibrosis in response to many pathogenic stimuli. Consequently, it is widely accepted that a better understanding of how the PDGF signaling cascade is regulated under these conditions will likely aid in the development of new therapeutic targets for liver fibrosis. 
A central focus of the current study is the scaffold protein, synectin, which links the transport of cell-surface receptors to their intracellular signaling pathways. Synectin (also referred to in the literature as GIPC) contains a PDZ domain that mediates the binding of multiple transmembrane proteins either directly or through coreceptors, such as the glycoprotein neuropilin-1 (NRP-1) (8, 9). Previous work showed that synectin, with its NRP-1 binding partner, played a key role in fibronectin fibril assembly in tumor-associated myofibroblasts (9), raising the possibility that a similar phenomenon may take place in broader pathological conditions such as liver fibrosis; this is a phenomenon, however, that awaits experimental confirmation. Moreover, the effects that synectin has on the regulation of PDGFRs and their downstream fibrogenic response have not been examined, leading us to the present study aimed at investigating the role of this protein on PDGFR signaling and cellular functions in HSC.

We report that synectin levels, along with PDGFR levels, are elevated in liver tissue derived from patients affected by cirrhosis, leading us to hypothesize that these proteins are components of a seamless pathway that plays a role in fibrogenic responses. To stringently test this hypothesis, we used an in vivo model of chronic liver injury performed in mice genetically engineered to carry a deletion of this gene in HSC. Parallel mechanistic experiments were also performed in isolated HSC in which the levels of synectin had been depleted using specific shRNAs. Combined, these studies revealed that synectin inactivation in HSCs decreases the levels of PDGFRs by complementing transcriptional and posttranscriptional mechanisms, alters the genome-wide expression of other important profibrogenic gene expression networks, and protects against hepatic fibrosis. Therefore, the findings derived from these investigations establish a previously unknown role for synectin in the regulation of the PDGF pathway, which is critical for the development of liver fibrosis. This raises the possibility that synectin may become an attractive target for the development of future therapeutic approaches to treat a variety of common liver diseases.

\section{Results}

Synectin deletion from HSC attenuates hepatic fibrogenesis in vivo. Synectin is known to play a key role in soluble fibronectin deposition in cancer-associated fibroblasts through an interaction with NRP-1 (9). This was of particular interest, as it has been previously shown that NRP-1 is upregulated in cirrhosis and inhibition of NRP-1 in HSC was protective against liver fibrosis in a murine model (6). Given the known interaction between NRP-1 and synectin and the presence of synectin with HSC, we sought to determine if synectin deletion from HSC in vivo would prevent fibrosis development in a chemical model of liver fibrosis. Although synectin is detected in multiple liver cell types, PDGFR expression is selective to HSC (10) (Supplemental Figure 1, A-C; human cell lines and primary murine liver cells; supplemental material available online with this article; https://doi.org/10.1172/jci.insight.92821DS1); based on this, we focused on the role of synectin in HSC, which is the cell type responsible for fibrogenesis. To accomplish this aim, mice carrying floxed synectin alleles were crossed with animals that express Cre under the Col1A1 promoter to generate mouse lines with selective deletion of synectin from cells expressing Col1A1, which — in liver tissue - is specific of HSC, herein referred to as $\mathrm{Col}^{\text {cre }} /$ Synectin ${ }^{\mathrm{f} / \mathrm{fl}}$. HSC isolated from Synectin ${ }^{\mathrm{f} / \mathrm{fl}}$ mice demonstrated reduction in synectin and both PDGFR isoforms upon in vitro transduction with AdCre-eGFP compared with AdLacZ (Supplemental Figure 1D). Subsequently, we used the $\mathrm{CCl}_{4}$ liver injury model to induce liver fibrosis as previously described (6). Isolated $\mathrm{HSC}$ from $\mathrm{CCl}_{4}$-treated mice showed striking increases of both PDGFRs and synectin expression in the $\mathrm{CCl}_{4}$-treated group (Supplemental Figure 1E). Using quantitative PCR (qPCR), we found a significant increase in collagen expression in the whole liver of Synectin ${ }^{\mathrm{f} / \mathrm{fl}}$ mice, which was attenuated in $\mathrm{Co}^{\text {cre }} /$ Synectin ${ }^{\mathrm{fl} / \mathrm{fl}}$ mice (Figure 1A). We also detected lower collagen deposition in the $\mathrm{Col}^{\text {cre }} /$ Synectin $^{\mathrm{f} / \mathrm{fl}}$ mice, as measured by hydroxyproline assay (Figure $1 \mathrm{~B}$ ), Sirius red staining, and immunofluorescence microscopy (Figure 1C and Supplemental Figure 2A). Immunofluorescence confocal laser microscopy demonstrated a reduction in the protein levels of PDGFR- $\alpha$, PDGFR- $\beta$, and $\alpha$-smooth muscle actin ( $\alpha$-SMA) in $\mathrm{Co}^{\text {cre }} /$ Synectin ${ }^{\mathrm{fl} / \mathrm{fl}}$ mice treated with $\mathrm{CCl}_{4}$ compared with their Synectin ${ }^{\mathrm{fl} / \mathrm{fl}}$ littermates (Figure 1C, quantitation in Supplemental Figure 2, B-E). Western blot analysis of whole liver lysates after 6 weeks of $\mathrm{CCl}_{4}$ injection showed an attenuation in the upregulation of PDGFR- $\alpha$ in the $\mathrm{Col}^{\text {cre }} /$ Synectin ${ }^{\mathrm{fl} / \mathrm{fl}}$ when compared with Synectin ${ }^{\mathrm{f} / \mathrm{fl}}$ mice (Figure $1 \mathrm{D}$ quantitation in Supplemental Figure 2F). Similar findings were noted for PDGFR- $\beta$ and synectin (Figure 1D).

Next, we studied the role of synectin in a bile duct ligation (BDL) model to test our hypothesis in a complementary cholestatic-type liver injury. Similar to our finding in the $\mathrm{CCl}_{4}$ model, we found significant increases in collagen mRNA by qPCR, collagen deposition by hydroxyproline, collagen immunostaining, 
A
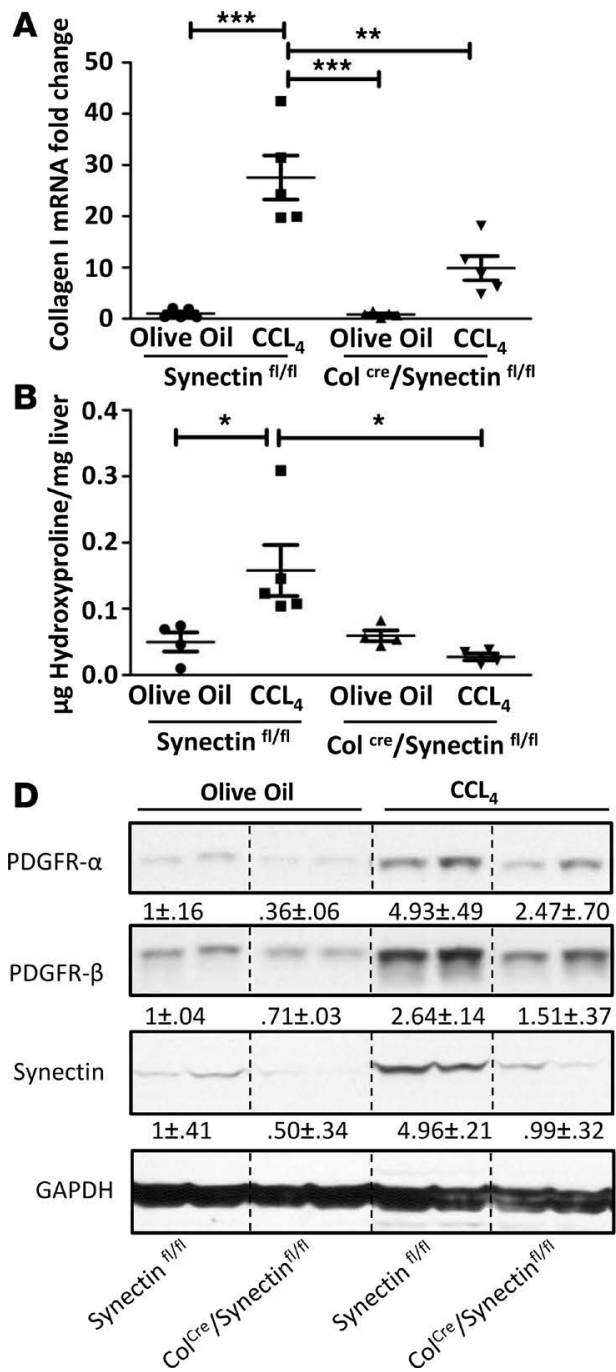

C

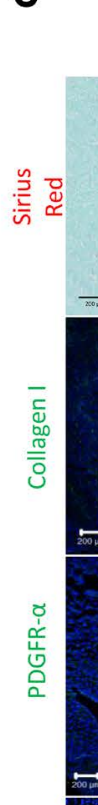

Olive Oil

$\overline{\text { Synectin }^{\mathrm{fl} / f \mathrm{fl}} \quad \text { ColCre}^{\mathrm{C}} \text { Synectin }^{\mathrm{fl} / f l}}$

$\frac{\mathrm{CCL}_{4}}{\text { Synectin }^{\mathrm{fl} / f l} \quad \mathrm{Col}^{\text {Cre }} / \text { Synectin }^{\text {fl/fl }}}$

Figure 1. Synectin deletion from HSC attenuates hepatic fibrogenesis in vivo. Mice with HSC selective deletion of synectin (Colcre/Synectin ${ }^{\text {fl/fl) }}$ and their control littermates (Synectin ${ }^{\text {fl/fl) }}$ were treated with either olive oil (vehicle) or CCI $_{4}$ via i.p. injections twice a week for 6 weeks. The livers were then harvested and prepared for analysis through isolation of mRNA or protein, or fixation of liver tissue for immunostaining and Sirius red analysis. (A) qPCR for collagen-1 mRNA levels showed a significant reduction in Colcre/Synectin ${ }^{\mathrm{fl} / \mathrm{fl}}$ mice liver after $\mathrm{CCl}_{4}$ injection, $n=4-7$ per group. (B) Collagen content was reduced in Colcre/ Synectin ${ }^{\text {fl/fl }}$ mice after $\mathrm{CCl}_{4}$ injection, as demonstrated by assessing hepatic hydroxyproline assay, $n=4-7$ per group. (C) Liver sections ( $5 \mu$ m) were stained with Sirius red to represent the fibrotic strands correlating with the degree of fibrosis. Additional liver sections were stained with antibodies against the fibrotic proteins collagen-1, PDGFR- $\alpha$, PDGFR- $\beta$, and $\alpha$-SMA (green) in conjunction with nuclear costaining with DAPI (blue), representative images shown. Staining revealed decreased expression of collagen-1, PDGFR- $\alpha$, PDGFR- $\beta$, and $\alpha$-SMA in Col ${ }^{\text {cre }} /$ Synectin $^{\text {fl/fl }}$ mice after CCI $_{4}$ injection compared with control littermates. Quantitation was performed using Imagej, with fold change displayed on the micrographs and graphs located in Supplemental Figure 2 , A-E; $n=$ 3-5. Scale bars: $200 \mu \mathrm{m}$. (D) Lysates from whole mouse liver were used to assess protein levels of PDCFR- $\alpha$, PDCFR- $\beta$, and synectin in Colre/Synectin ${ }^{\text {fl/fl }}$ mice after $\mathrm{CCl}_{4}$ injection. Samples were run on the same gel but were noncontiguous. $n=3-4$. Quantification was performed using Image), with the densitometric values displayed below the blots and graphs located in Supplemental Figure $2 \mathrm{~F}$. All data are displayed as mean $\pm \mathrm{SEM}\left({ }^{*} P<0.05\right.$ ). Each dot in the scatter plot indicates an individual animal in each of the panels. One-way ANOVA with Bonferroni's multiple comparison tests were used to analyze groups for statistical significance $\left({ }^{*} P<0.05,{ }^{* *} P<0.001,{ }^{* *} P<0.0001\right)$.

and increased PDGFR- $\alpha$ and $-\beta$ by Western blot in Synectin ${ }^{\mathrm{f} / \mathrm{fl}}$ mice undergoing BDL compared with sham surgery littermates. These changes were attenuated in the $\mathrm{Col}^{\mathrm{cre}} /$ Synectin $^{\mathrm{f} / \mathrm{fl}}$ mice (Supplemental Figure 3 , A-D). Thus, genetic experiments in mice using 2 well-validated models of fibrogenesis reveal that synectin is required for PDGFR upregulation in the development and progression of hepatic fibrogenesis.

Knockdown of synectin in HSC results in the downregulation of canonical profibrogenic gene expression networks via histone modifications and $p 300$. To gain insight into molecular mechanisms by which the genetic inactivation of synectin impacts on liver fibrosis, we performed next-generation sequencing of mRNA (mRNASeq) isolated from human HSC (hHSC) in which the levels of synectin were significantly reduced using shRNA-mediated knockdown. A heatmap (ClustVis) (11) of the top 464 regulated genes (as selected by 

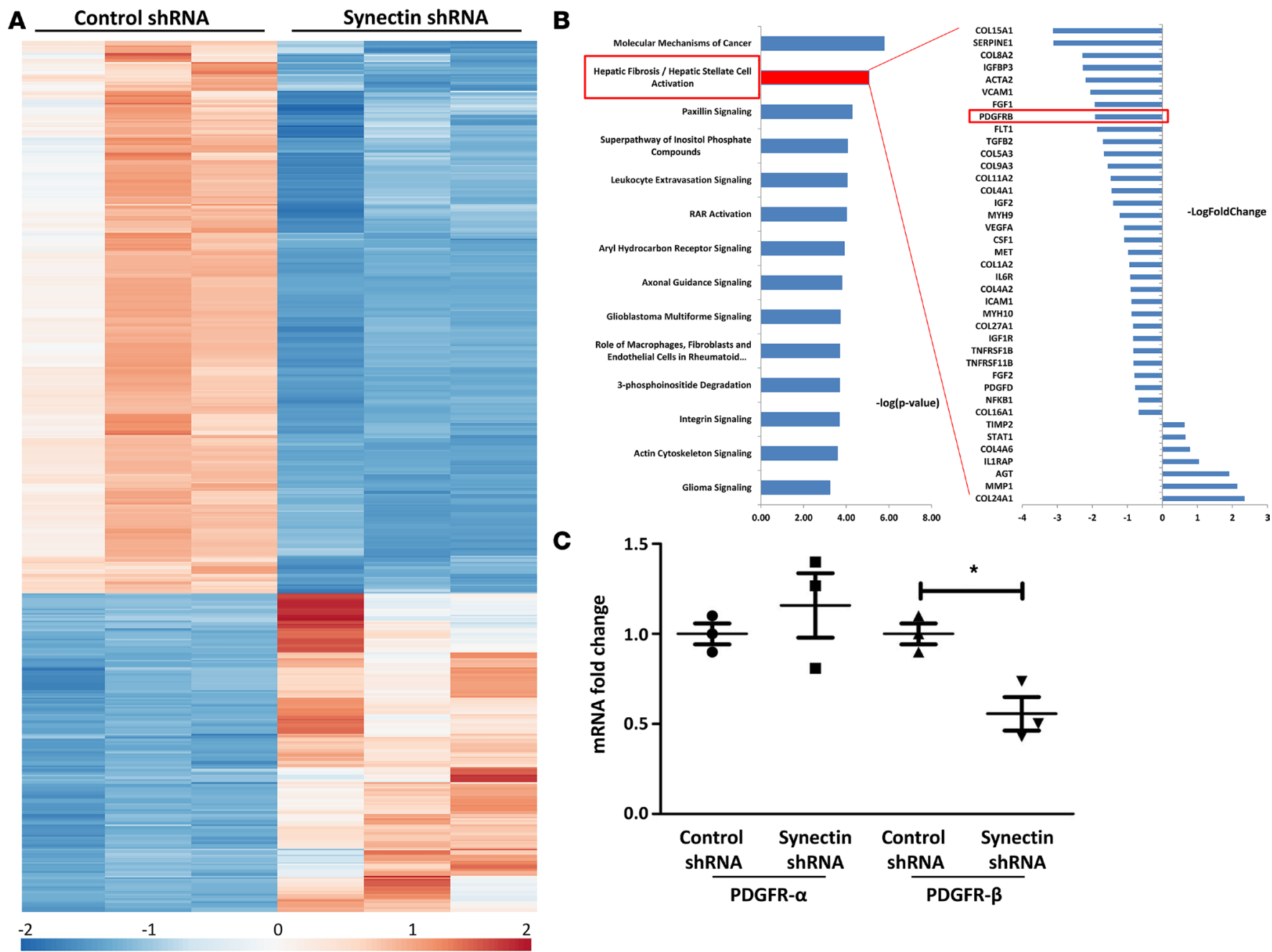

Figure 2. The HSC activation pathway is highly regulated by synectin through transcriptomic analysis by NextGen sequencing (mRNA-Seq). (A) Heatmap of whole genome gene expression depicting differences in the expression profile between control and synectin-knockdown human HSC (hHSC) of all genes with a logFC $>1.5$ of $<-1.5$. (B) Ingenuity pathway analyses (IPA, Qiagen) showed that the fibrotic pathway was differentially regulated between the groups. FDR $<0.05, P<0.05$. Regulation of specific genes within the hepatic fibrosis pathway are shown, with PDGFR- $\beta$ highlighted. (C) qPCR from hHSC with and without synectin knockdown demonstrated a reduction in PDGFR- $\beta$ mRNA. Results are from 3 independent experiments. Student's unpaired, 2 -tailed $t$ test was used to analyze the differences between groups for statistical significance $\left({ }^{*} P<0.05\right)$.

absolute value of $\log$ fold change $(\log \mathrm{FC})>1.5 ; \mathrm{FDR}<0.05$ and $P<0.05)$ analyzed in mRNA-Seq provided visualization of distinct patterns of gene regulation between the control and synectin-knockdown HSC (Figure 2A and Supplemental Table 1). Ingenuity pathway analyses (IPA, Qiagen) demonstrate downregulation of mRNA of the genes from the Fibrosis/HSC Activation pathway in synectin-knockdown cells, which scores second only to Molecular Mechanism of Cancer pathway (Figure 2B and Supplemental Table 2). Pictorial visualization for the PDGF signaling pathway showed multiple downregulated genes (depicted in green) in synectin-depleted HSC (Supplemental Figure 4A). PDGF ligands (A, $\mathrm{B}, \mathrm{C}$, and D isoforms) were analyzed from the RNA-Seq data set and showed no significant differences between control cells and synectin-knockdown cells and low expression levels compared with PDGFR- $\beta$ (Supplemental Figure 4B). Compared with control cells, those carrying a knockdown in synectin show the most significant downregulation in the genes encoding for PDGFR- $\beta$, TGFB2, IGFBP3, FGF1, PDGF-D, Serpine1, FLT1, VEGF-A, and many types of collagens. We also used qPCR to confirm the RNA-Seq results showing that PDGFR- $\alpha$ mRNA was unchanged after synectin knockdown, whereas PDGFR- $\beta$ mRNA was significantly downregulated under these conditions (Figure $2 \mathrm{C}$ ). Thus, these combined analyses demonstrate that the decrease of fibrosis observed upon the genetic inactivation of synectin results primarily in affecting the regulation of profibrogenic gene networks, a result that is striking considering 
A

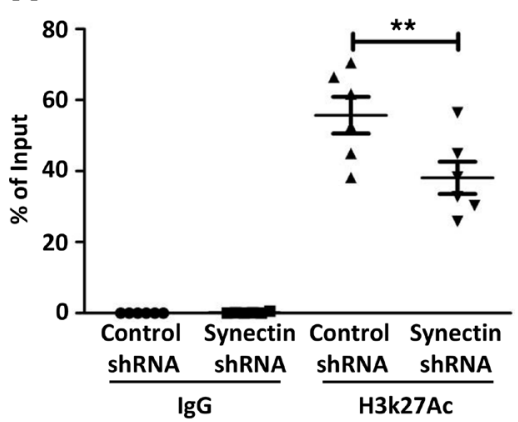

B

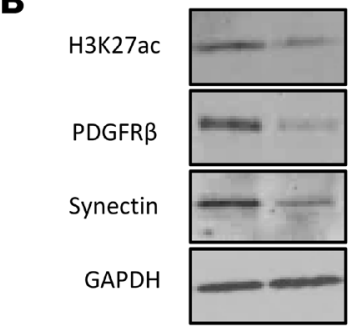

Synectin shRNA

C

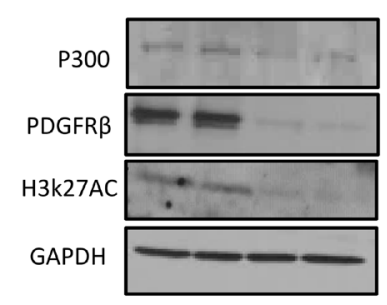

P300 ShRNA - -++
D

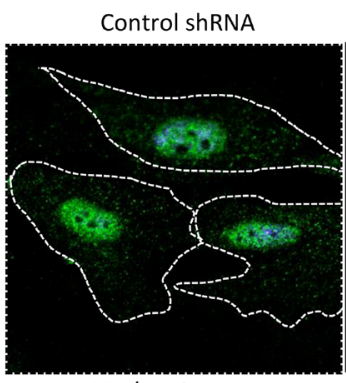

P300/Dapi
Synectin shRNA

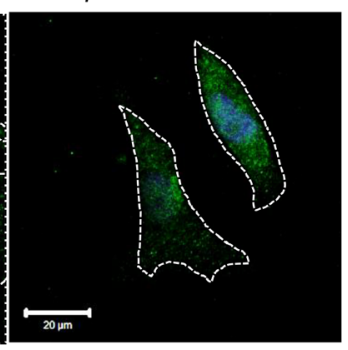

E

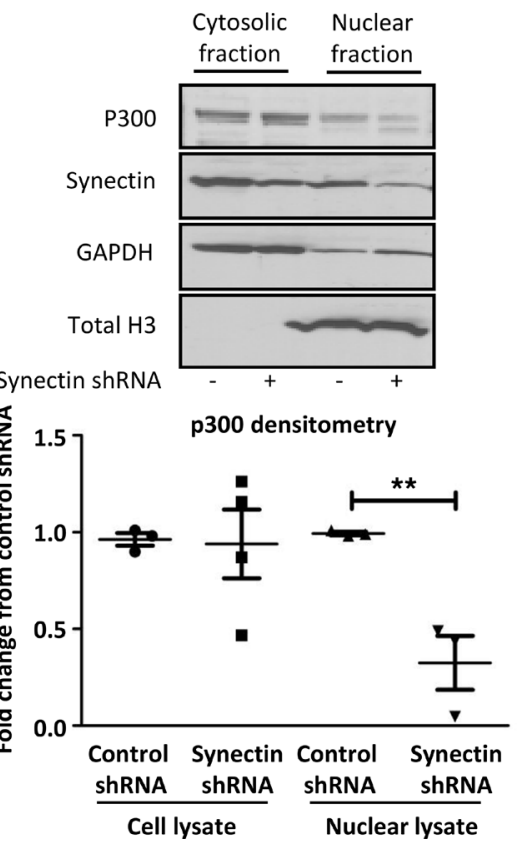

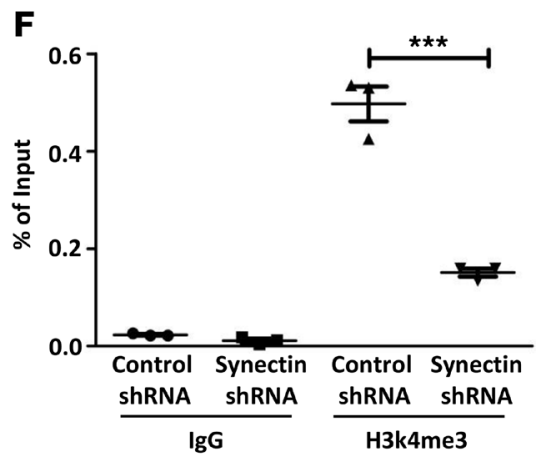

G

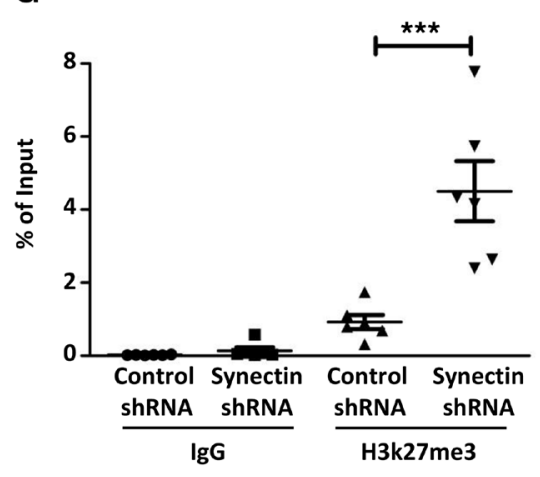

Figure 3. Knockdown of Synectin in HSC results in the downregulation of canonical profibrogenic gene expression networks via histone modifications and p300. (A and B) The histone modification H3K27ac is associated with activation of gene expression and was measured at the PDCFR- $\beta$ gene locus by ChIP using H3K27ac antibody (A) and Western blot (B). A reduction in acetylation of histone H3 at lysine 27 was observed in synectin-knockdown hHSCs compared with control cells, $n=6$ (A), $n=3$ (B). The efficiency of shRNA-mediated knockdown of synectin was also shown. (C) shRNA-mediated knockdown of p300 decreased the protein expression of PDGFR- $\beta$ and H3K27ac in hHSCs as shown by Western blot, $n=3$. (D) hHSC were stained using p300 antibody (green) with background DAPI stain to show nucleus (blue). Decreased nuclear localization of p300 was observed in synectin-knockdown hHSCs. White broken line was used to define the outline of the cell. (E) Cell lysates and nuclear fractions from synectin-knockdown hHSC showed a reduction in p300 protein levels by Western blot in the nuclear fraction only. Densitometry was analyzed using Image) and is depicted in the graph below, $n=3$. (F and $\mathbf{G}$ ) ChIP using H3K4me3 and H3K27me3 antibodies showed decreased methylation of histone $\mathrm{H3}$ at lysine 4 and increased methylation of histone $\mathrm{H3}$ at lysine 27 of PDGFR- $\beta$ promoter after synectin knockdown, further indicative of repressed gene transcription. All data are expressed as mean \pm SEM. Scale bar: $20 \mu \mathrm{m}$. One-way ANOVA with Bonferroni's multiple comparison test were used to analyze groups for statistical significance $\left({ }^{* *} P<0.001,{ }^{* *} P<0.0001\right)$.

that the main role of this protein is related to vesicular transport. Therefore, we next tested the hypothesis that synectin regulates HSC-mediated fibrosis through its effect on PDGF receptors.

Given the imperative role of chromatin-mediated events on gene regulation, we monitored the type of histone marks on chromatin associated with PDGFR- $\beta$. First, we assessed the role of synectin knockdown on specific histone modifications associated with the PDGFR- $\beta$ promoter using antibodies against histone 3 lysine 27 acetylation (H3K27ac). H3K27ac is known as one of most important modifications to the chromatin packaging protein $\mathrm{H} 3$, which involves the acetylation at the 27th lysine residue of this histone protein. It is usually associated with the higher activation of transcription as an active enhancer mark. We found that, under synectin-knockdown conditions, the occupancy of the PDGFR- $\beta$ promoter by $\mathrm{H} 3 \mathrm{~K} 27 \mathrm{ac}$ was reduced, suggesting that this was a mechanism of chromatin remodeling for silencing this gene (Figure 3A). Furthermore, upon synectin knockdown, the total cell H3K27ac was reduced (Figure 3B and Supplemental Figure 5A). To gain insight into molecular mechanisms that mediated this effect, we examined p300, which is known to acetylate H3 at K27. In this regard, we found that, 
A

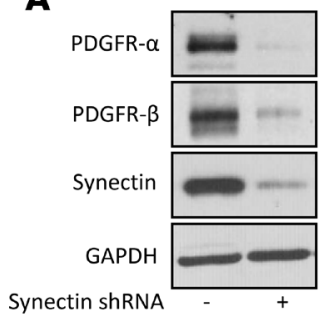

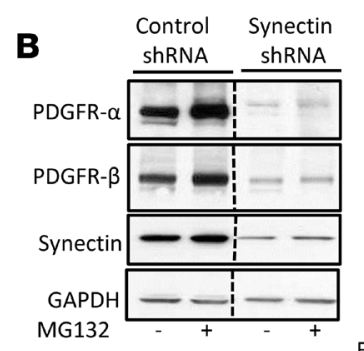
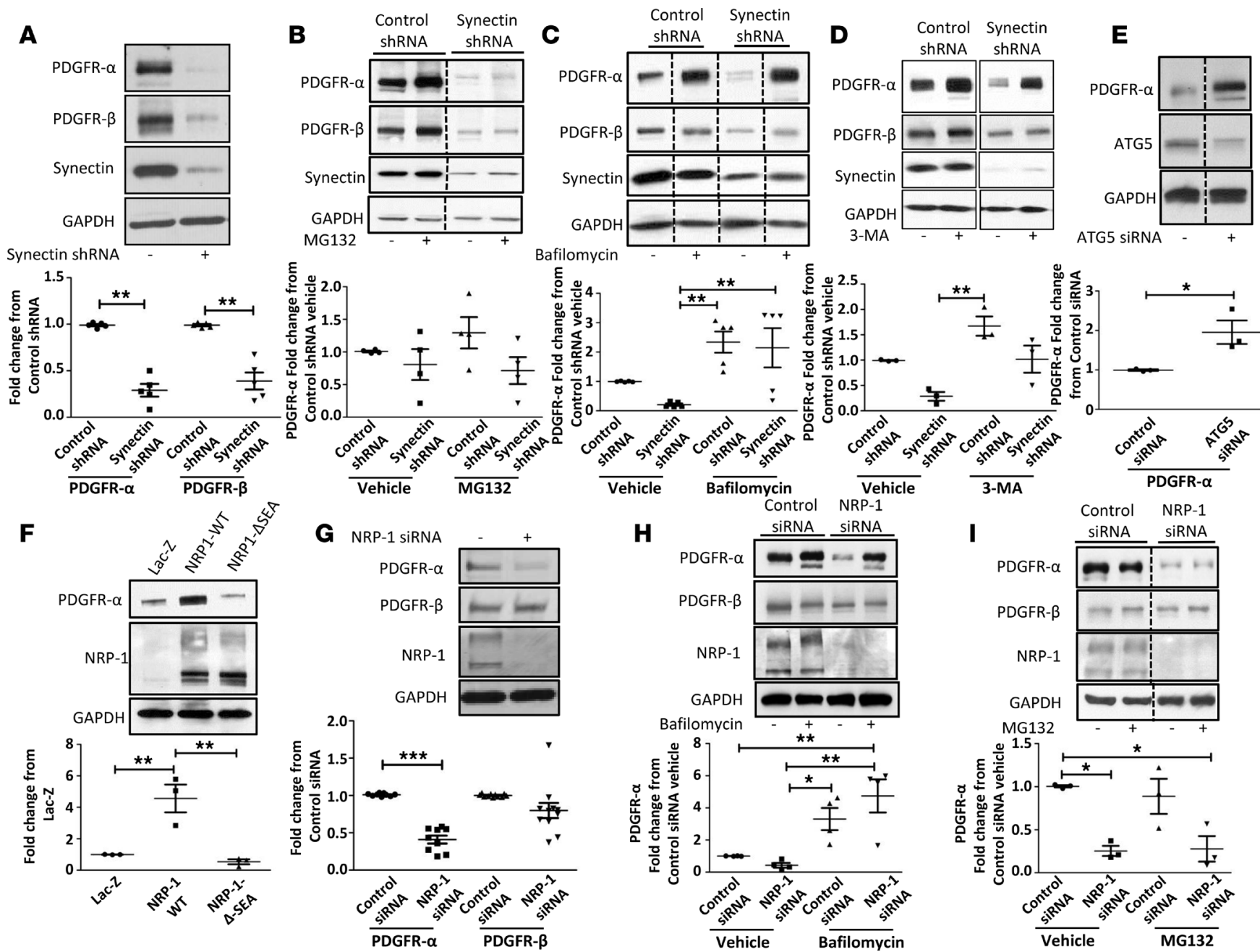

ATG5 SiRNA - + +
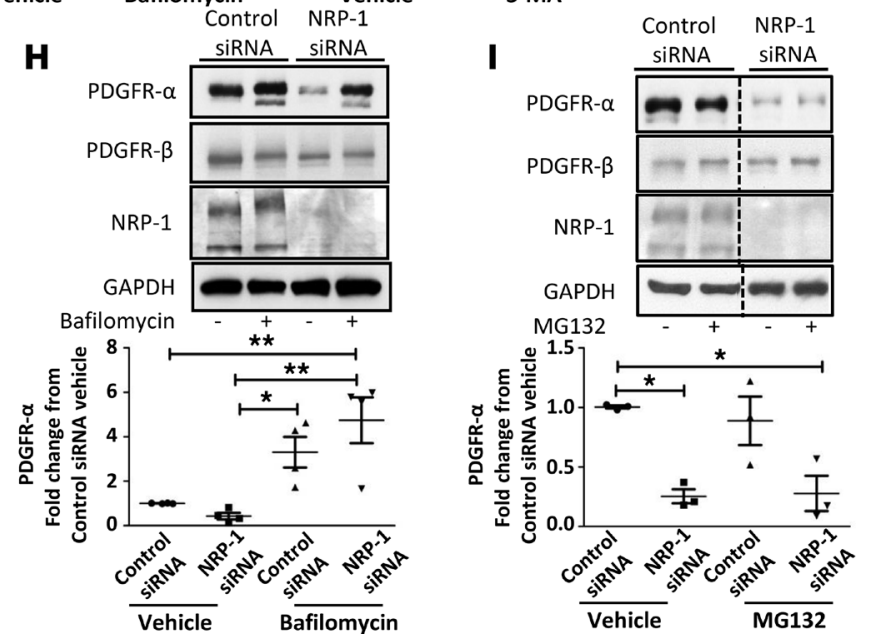

Figure 4. Synectin regulates PDGFR protein levels. (A) shRNA-mediated synectin knockdown in hHSC decreases both PDGFR- $\alpha$ and PDGFR- $\beta$ protein levels as depicted by Western blot. Densitometry located in the graph below, $n=5$. (B) Control or synectin-knockdown hHSCs were treated with the proteosomal inhibitor MG132 $(25 \mu \mathrm{M})$ for 4 hours; however, in PDGFR- $\alpha$ or $-\beta$, protein levels were not increased by proteosomal inhibitor MG132 ( $n=3)$ in hHSC with synectin knockdown. (C and $\mathbf{D})$ In contrast, PDCFR- $\alpha$ levels were increased by inhibition of autophagy by bafilomycin (10 $\mu \mathrm{M}$, overnight) and 3-MA (1 $\mu$ M, overnight) in both control and synectin-knockdown hHSC, $n=3$. (E) Inhibition of autophagy by ATG5 siRNA increased PDCFR- $\alpha$ protein levels in hHSCs, $n=3$. (F) LX-2 cells were transfected with a LacZ control plasmid, a plasmid encoding WT NRP-1 (NRP-1-WT), or a plasmid encoding a NRP-1 mutant lacking the SEA domain (NRP-1- $\triangle$ SEA), and PDGFR- $\alpha$ protein levels were determined by Western blot. Overexpression of WT NRP-1 enhanced PDGFR- $\alpha$ expression; however, the NRP-1 mutant resulted in decreased PDGFR- $\alpha$ protein levels, $n=3$. (C) siRNA-mediated NRP-1 knockdown in hHSC showed reduction in PDGFR- $\alpha$ protein levels without affecting PDGFR- $\beta$ protein levels as shown by Western blot, $n=7$. (H) Bafilomycin (10 $\mu$ M) treatment increased PDGFR- $\alpha$ protein levels following siRNA-mediated NRP-1 knockdown as observed by Western blot, $n=3$. (I) hHSCs were transfected with NRP-1 siRNA or a control, followed by treatment with MG132 (25 $\mu$ M). PDGFR- $\beta$ protein levels were not increased by MG132 treatment in NRP-1-knockdown cells, $n=3$. Samples were run on the same gel but were noncontiguous in B-E and I. Data are expressed as mean \pm SEM. Densitometry for all experiments was analyzed by Image) and are depicted below their respective blots. One-way ANOVA with Bonferroni's multiple comparison test was used to analyze groups for statistical significance $\left({ }^{*} P<0.05,{ }^{* *} P<0.001,{ }^{* *} P<0.0001\right)$.

upon knockdown of p300 by shRNA, there was a reduction of the H3K27ac levels with a concomitant decrease in PDGFR- $\beta$ protein levels (Figure 3C and Supplemental Figure 5B). This result was congruent with the additional observation that cells carrying a synectin knockdown displayed a reduction in the nuclear levels of p300, as detected using immunofluorescence and Western blot analyses (Figure 3, D and E). Further ChIP studies were performed on the methylation pattern of the PDGFR- $\beta$ promoter: the H3K27me3 modification, which in many cases antagonizes H3K27ac, and another enhancer mark H3K4me3. This ChIP analysis showed a reduction in the stimulatory H3K4me3 mark and an increase in the inhibitory H3K27me3 mark (Figure 3, F and G), which is congruent with our H3K27ac data and suggests a transition of euchromatin to heterochromatin for silencing of the PDGFR- $\beta$ gene in response to synectin knockdown. 
Synectin regulates PDGFR protein levels. Given the observed changes in PDGFR- $\beta$ at the transcription level, we sought to confirm changes in PDGFR- $\beta$ at the protein level. First, to gain a more global assessment of the effect of synectin depletion, we used a receptor tyrosine kinase protein array to determine if not only PDGFR- $\beta$, but also other similar receptors, might be regulated at the protein level. The protein array confirmed the downregulation of the PDGFR- $\beta$ in synectin-knockdown HSC and also showed a similar pattern of downregulation of PDGFR- $\alpha$ (Supplemental Figure 6). It further confirmed the PDGFR isoforms as interesting targets for further study. In vitro Western blot experiments aimed at confirming the results of mRNA-Seq, protein array, and studies from cirrhotic murine liver tissue revealed that synectin knockdown resulted in decreased levels of the PDGFR- $\beta$ protein and PDGFR- $\alpha$ protein (Figure 4A). While this is consistent with the decrease in both proteins observed in response to fibrosis induction in mice lacking synectin in HSC (Figure 1D) and the protein array (Supplemental Figure 6), only PDGFR- $\beta$ and not $-\alpha$ was affected in our mRNA-Seq studies and corroborative qPCR analysis (Figure 2, B and C). Thus, it appeared that the 2 PDGFR isoforms are regulated by synectin through different mechanisms. This observation led us to examine whether the decrease in the levels of PDGFR- $\alpha$ protein upon synectin knockdown was the result of its degradation. Using pharmacological approaches, we ruled out a role for either proteasomal or lysosomal degradation pathways in the regulation of PDGFR- $\alpha$ protein levels (Figure 4B). However, upon synectin knockdown, PDGFR- $\alpha$ protein levels were increased by inhibition of autophagy by 3-MA or bafilomycin (Figure 4, C and D), both in the presence or absence of PDGF (Supplemental Figure 7A). Additionally, ATG5 siRNA, which inhibits autophagy, increased PDGFR- $\alpha$ protein levels (Figure 4E). In contrast, inhibition of autophagy failed to increase PDGFR- $\beta$ protein levels after synectin knockdown (Figure 4, C and D, and Supplemental Figure 7B).

Next, we sought to determine whether disrupting the interaction of synectin with the PDGFR coreceptor and synectin binding partner NRP-1 had a similar effect to synectin knockdown. For this purpose, we overexpressed a NRP-1 mutant that lacked the SEA domain (NRP-1 $\triangle \mathrm{SEA}$ ), as this protein is unable to bind synectin (9). For control purposes, we overexpressed WT NRP-1. Using this approach, we detected a significant increase in the levels of PDGFR- $\alpha$ protein with overexpression of WT NRP1 , which did not occur with the overexpression of NRP-1 $\triangle$ SEA (Figure $4 \mathrm{~F}$ ). This result suggests that synectin binding to NRP-1 is important for the protein stability of PDGFR- $\alpha$. Additionally, knockdown of NRP-1 by siRNA also resulted in decreased PDGFR- $\alpha$ protein levels (Figure 4G). Furthermore, the effects of NRP-1 knockdown on PDGFR- $\alpha$ were attenuated by inhibition of the autophagy pathway, but not by blockade of the proteasomal or lysosomal degradation pathways (Figure 4, H and I). Interestingly, while synectin knockdown altered both PDGFR- $\alpha$ and $-\beta$ protein levels, NRP-1 knockdown altered only PDGFR- $\alpha$ and not PDGFR- $\beta$ protein levels (Figure 4G), indicating that synectin may have broader effects on fibrogenesis than NRP-1.

PDGFR- $\alpha$ undergoes selective autophagy and degradation via the ubiquitination of specific lysine residues. Our studies show that, in contrast to PDGFR- $\beta$, PDGFR- $\alpha$ is not regulated at the transcriptional level but rather through autophagic degradation upon synectin knockdown. In this regard, IP studies demonstrated an association of the autophagy receptor p62 with PDGFR- $\alpha$ (Figure 5A). Confocal microscopy was also performed with and without bafilomycin, as a means to prevent the fusion of the autophagosome with the lysosome, which would allow us to detect the localization of protein complexes within the autophagosome prior to degradation (12). Interestingly, these experiments showed greater colocalization of PDGFR- $\alpha$ with p62 when compared with PDGFR- $\beta$, both in the absence of bafilomycin and in the presence of bafilomycin, supporting our biochemical data (Figure 5B). We also determined whether the autophagic receptor p62 associated to the PDGFR- $\alpha$ protein after synectin knockdown. IP of the PDGFR- $\alpha$ after synectin knockdown revealed an increased binding of p62 to PDGFR- $\alpha$ (Figure 5C). Finally, confocal microscopy of PDGFR- $\alpha$ after synectin knockdown showed increased colocalization with both LC3b and p62 (Figure $5, \mathrm{D}$ and $\mathrm{E})$. This data supports the hypothesis that PDGFR- $\alpha$ undergoes autophagic degradation by effectively interacting with the autophagy receptor $\mathrm{p} 62$.

Next, we sought to determine if p62 binding through ubiquitin accounted for the specific autophagic degradation of PDGFR- $\alpha$. To investigate this hypothesis, we examined the PDGFR- $\alpha$ and PDGFR- $\beta$ amino acid sequences focusing on 6 ubiquitin sites present in the cytosolic domain of PDGFR- $\alpha$ reported in the Prosite database (13). Two of the known ubiquitin sites within PDGFR- $\alpha$ (lysines 606 and 971) did not have equivalent lysine residues within PDGFR- $\beta$ (Figure 6A). Analysis of the PDGFR- $\alpha$ sequence from 7 species (Xenopus laevis, Gallus gallus, Homo sapiens, Mus musculus, Rattus norvegicus, Fugu rubripes, and Danio 
A

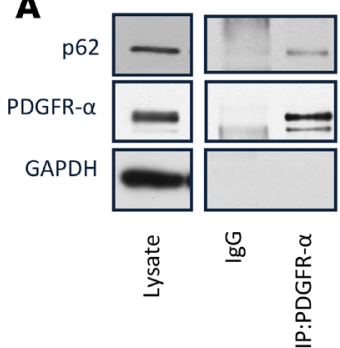

C

B

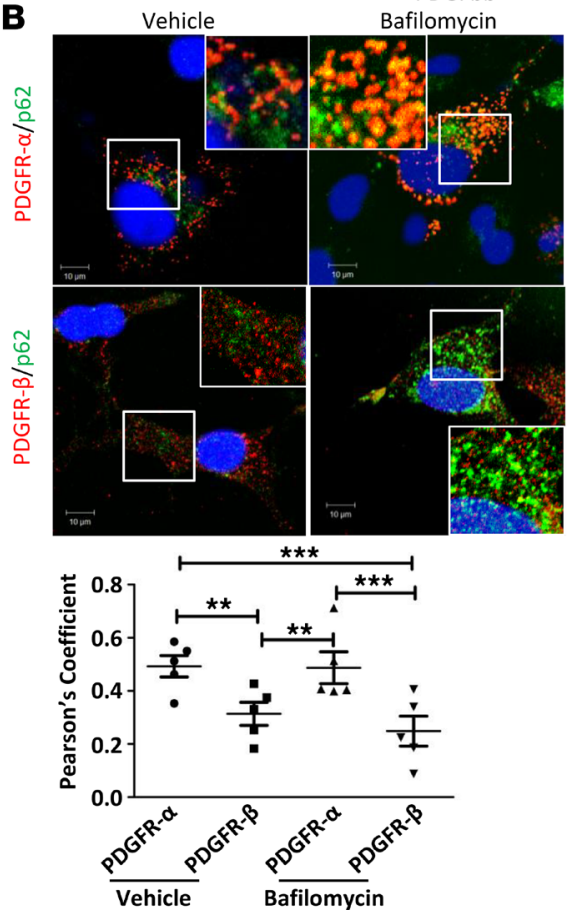

Lysate

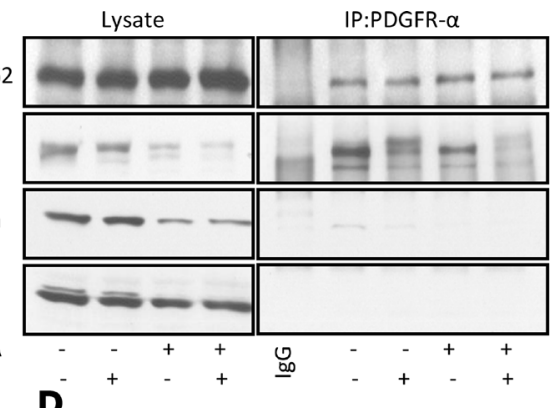

D

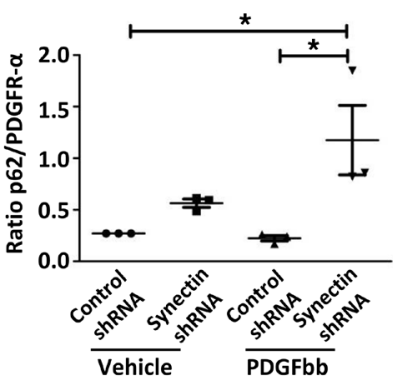

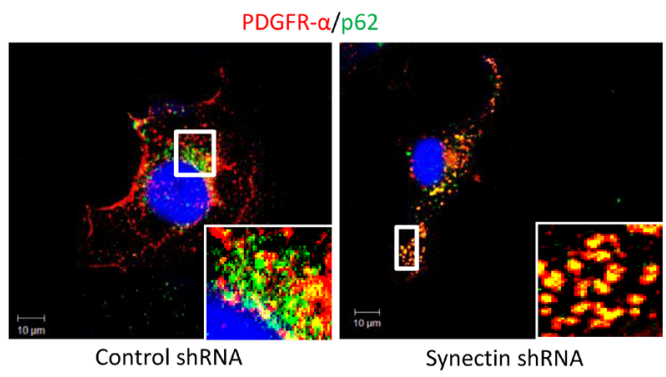

Synectin shRNA

E

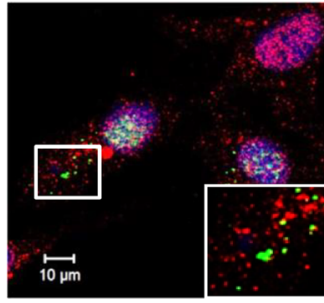

Control shRNA
PDGFR- $\alpha$ /LC3B-GFP

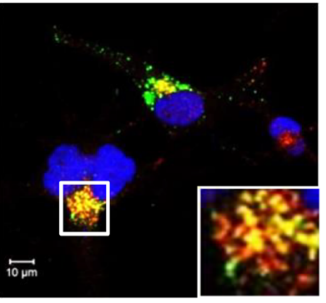

Synectin shRNA
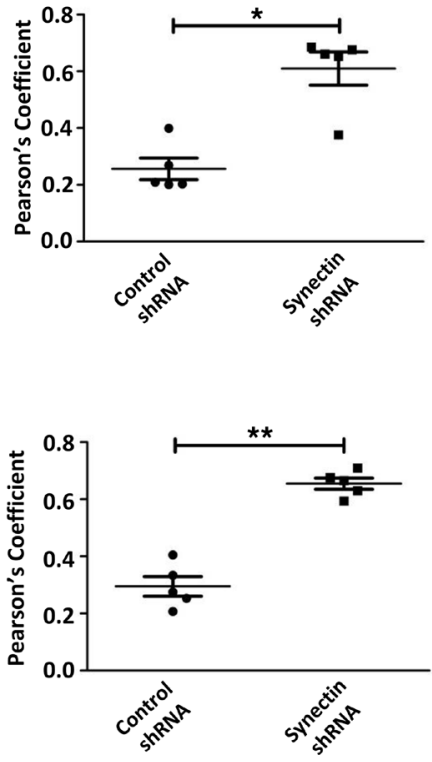

Figure 5. PDGFR- $\alpha$ undergoes selective autophagy and degradation. (A) Coimmunoprecipitation (Co-IP) was performed from hHSC lysates using an antibody against PDGFR- $\alpha$, and the recovered proteins were analyzed by Western blot, $n=4$. (B) hHSC were transduced with adenoviral flag-tagged PDGFR- $\alpha$ and PDGFR- $\beta$ constructs, followed by treatment with or without bafilomycin $(10 \mu \mathrm{M})$ and coimmunostained using flag and p62 antibodies. Imaging of hHSC showed colocalization of p62 with FLAG-PDGFR- $\alpha$, but not FLAG-PDGFR- $\beta$. Colocalization was measured using the Pearson's coefficient, calculated by JoCIP plug-in in Image, and it is displayed in the graph below. (C) Synectin was knocked down in hHSCs using shRNA, followed by treatment with PDGF-bb (10 ng/ $\mathrm{ml}$ ). Lysates were harvested and a Co-IP performed with an antibody against PDGFR- $\alpha$. The association between PDGFR- $\alpha$ and p62 increased in cells with synectin knockdown treated with PDCF-bb, as measured by increased ratio of p62 to PDGFR- $\alpha$ after synectin knockdown, $n=3$. (D) hHSC were transduced with adenoviral flag-tagged PDGFR- $\alpha$ construct and coimmunostained using flag and p62 antibodies. Representative pictures are shown. Colocalization was determined by measuring the Pearson's Coefficient calculated by JoCIP plug-in in Imagej, and it is displayed in the adjacent graph. (E) Synectin-knockdown cells were transduced with an adenoviral flag-tagged PDGFR- $\alpha$ construct and transfected with LC3b-GFP plasmid. Colocalization was determined by measuring the Pearson's coefficient calculated by JoCIP plug-in in Image, and it is displayed in the adjacent graph, $n=3$. For all colocalization experiments, 2 images were obtained from 3 separate wells for a total of 6 fields taken at $63 x$. All data are expressed as mean \pm SEM. Scale bars: $10 \mu \mathrm{m}$. Samples were run on the same gel but were noncontiguous in $\mathbf{A}$ and $\mathbf{C}$. One-way ANOVA with Bonferroni's multiple comparison test was used to analyze groups for statistical significance $\left({ }^{*} P<0.05,{ }^{* *} P<0.001,{ }^{* * *} P<0.0001\right)$. Student's unpaired $t$ test was used to analyze the differences between 2 groups ( ${ }^{*} P<0.05$, ${ }^{* *} P<0.001$ ).

rerio) showed that these lysine residues were highly conserved (Supplemental Figure 8A). Additionally, there was a high degree of sequence similarity between PDGFR- $\alpha$ and PDGFR- $\beta$ in the amino acids flanking lysine 606 and 971 (Figure 6A). Therefore, we mutated PDGFR- $\beta$ to introduce lysine residues (amino acids 606 and 971) at the sites that match the lysine residues in PDGFR- $\alpha$ (PDGFR- $\beta$ mutant 606/971). Overexpression of the PDGFR- $\beta$ mutant 606/971 in LX2 cells resulted in an increase of PDGFR- $\beta$ mutant after incubation with bafilomycin (Figure 6B). Overexpression of WT PDGFR- $\beta$ did not show any increase in its expression in the presence of bafilomycin, which is consistent with our findings with the endogenous protein (Figure 6B). Confocal microscopy of mutant PDGFR- $\beta$ 606/971 showed a significant increase in colocalization with LC3B and p62 when compared with WT PDGFR- $\beta$ (Figure 6, C and D). These data indicate that lysine 606 and/or 971 contribute to autophagic degradation of PDGFR- $\alpha$. Furthermore, we 
A

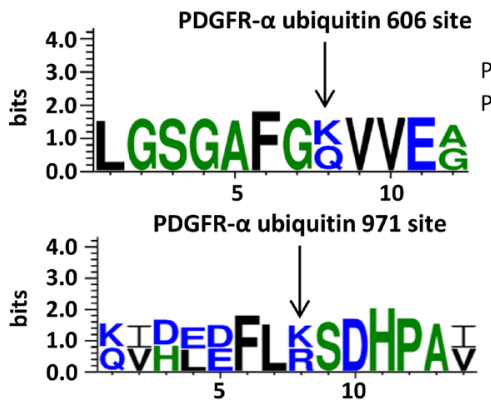

B

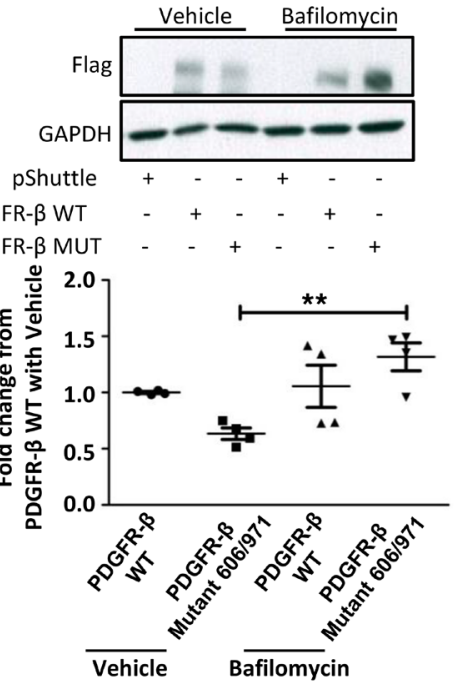

C
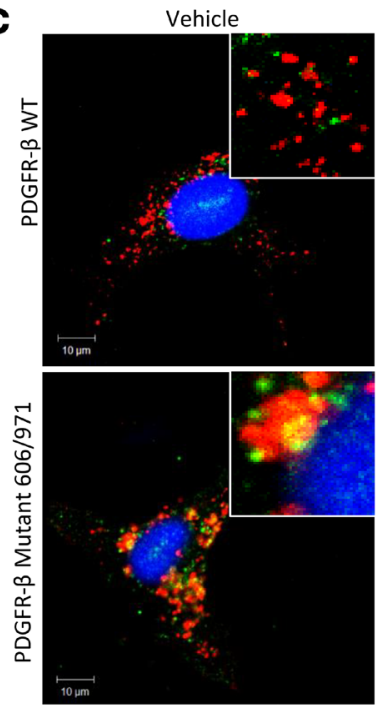

PDGFRß-LC3b
D PDGFR- $\beta$ WT

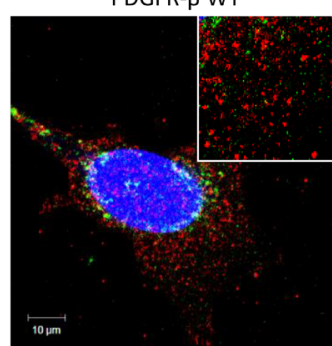

PDGFR $\beta-p 62$

E

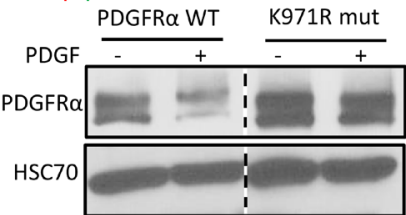

PDGFR- $\beta$ Mutant 606/971

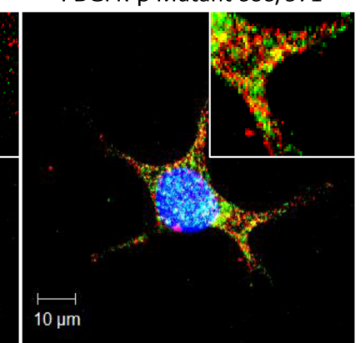

$10 \mu \mathrm{m}$

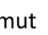


A

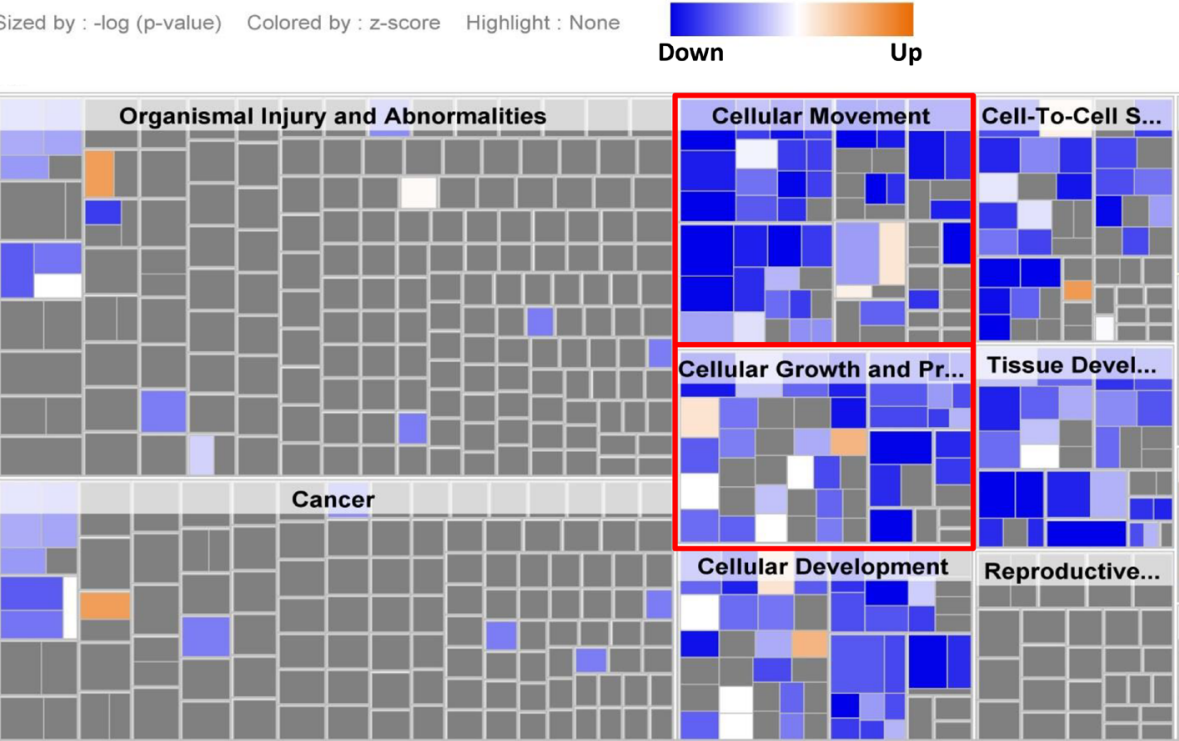

B

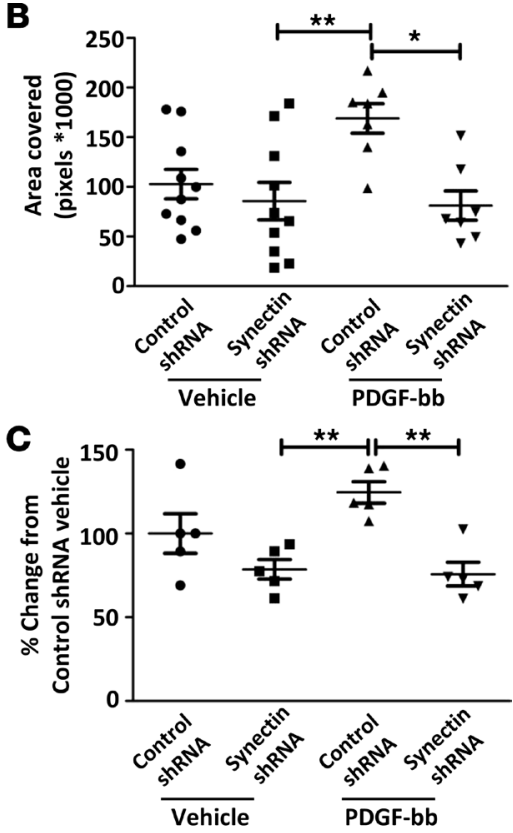

D

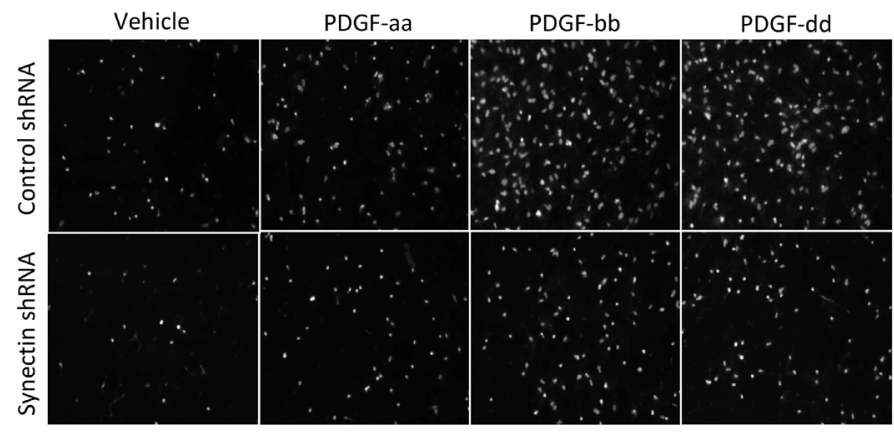

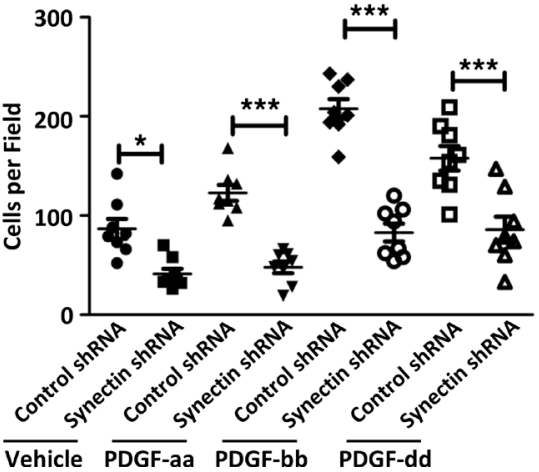

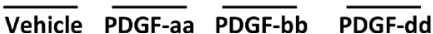

Figure 7. Synectin knockdown inhibits PDGF-stimulated migration and proliferation of HSC. (A) Ingenuity Pathway Analysis (IPA) of cellular function demonstrated a reduction in genes associated with cellular movement and proliferation in synectin-knockdown hHSC. (B and C) shRNA-mediated synectin knockdown in LX2 reduced migration as observed by scratch assay (B), and proliferation as measured by MTS assay in response to PDCF (C), $n=3$. For B, 3 images were obtained from each scratch, $n=3$. (D) Synectin expression was knocked down by shRNA (versus control shRNA) and migration assessed using a Boyden chamber in the presence of multiple PDGF ligands, $n=3$, magnification, 10x. The number of cells per field was quantified and is displayed in the adjacent graph. All data are expressed as mean \pm SEM of at least 3 independent experiments. One-way ANOVA with Bonferroni's multiple comparison test was used to analyze groups for statistical significance $\left({ }^{*} P<0.05,{ }^{* *} P<0.001,{ }^{* * *} P<0.0001\right)$.

7A,Supplemental Figure 9, and Supplemental Tables 2 and 3). To validate the IPA data, we examined SMA, collagen, and fibronectin in synectin-knockdown HSC; all 3 markers of stellate cell activation/fibrogenesis were decreased (Supplemental Figure 10, A-C). We next examined migration and proliferation in synectin-depleted HSC with PDGF-bb as a functional readout of HSC activation. We used a scratch assay for migration and MTS assay from proliferation. This experiment showed that the reduction in synectin protein levels results in a significant reduction in both proliferation and migration of HSCs (Figure 7, B and C). Similarly, HSC proliferation was diminished upon synectin knockdown by BrdU assay (Supplemental Figure 10D). We next used a peptide that blocks the PDZ domain of synectin (14) to determine if this also had inhibitory effects on migration. These studies, performed in a microfluidic flow chamber, found that the neutralizing peptide reduced HSC migration (Supplemental Figure 10E). We examined if the reduction in PDGFR phosphorylation, migration, and proliferation was due to changes in downstream signaling through AKT, but we found no significant difference in AKT activation (Supplemental Figure 11, A and B). However, knockdown of synectin did attenuate the increase of active Rac1 induced by PDGF (Supplemental Figure 11C). Similar observations were previously noted in HSC migration models of neuropilin, a synectin binding partner (6). We tested the other PDGF ligand isoforms (PDGF-aa and PDGF-dd) to determine if they had similar effects as PDGF-bb, as each ligand activates different receptor combinations, 
A

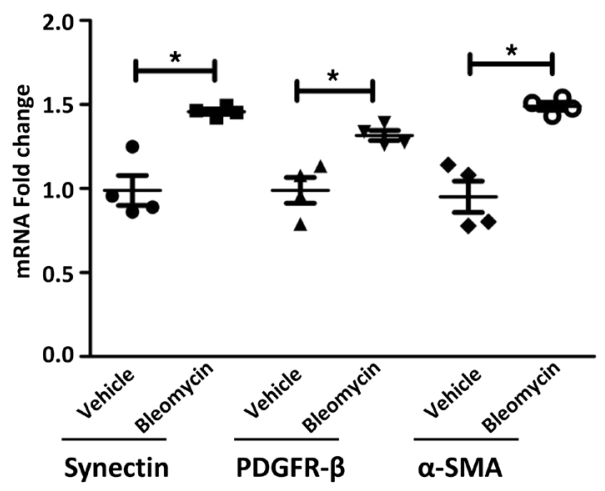

C

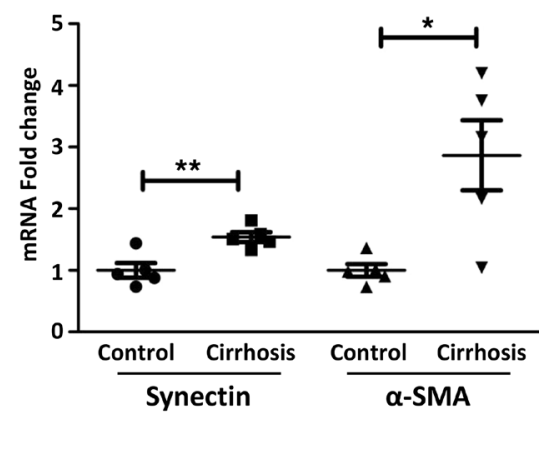

D

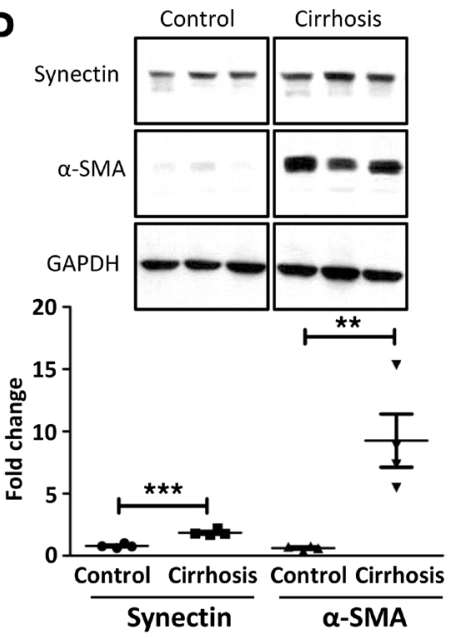

B

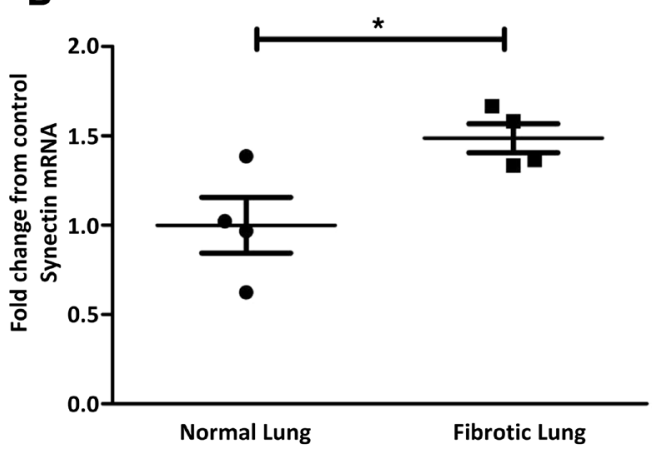

E

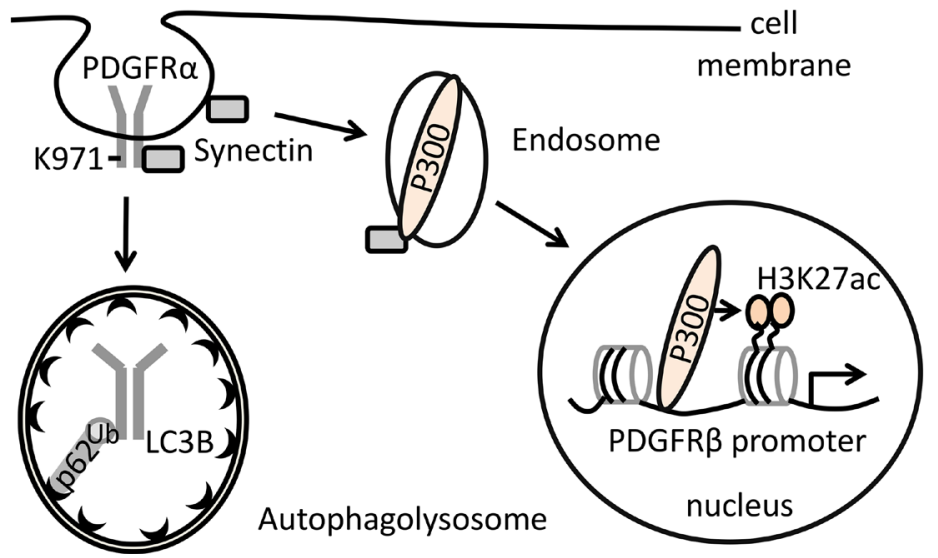

Figure 8. Synectin is upregulated in murine pulmonary fibrosis, human pulmonary fibrosis, and human cirrhosis. (A) mRNA was harvested from murine lungs treated with bleomycin to induce fibrosis or a vehicle control. qPCR was performed to analyze mRNA expression of synectin, PDCFR- $\beta$, or $\alpha$-SMA. We observed increased synectin, PDGFR- $\beta$, and $\alpha$-SMA mRNA in the bleomycin exposed samples, $n=3$. (B) Pulmonary fibroblasts were isolated from patients diagnosed with Idiopathic Pulmonary Fibrosis. mRNA was harvested from these fibroblasts and analyzed by qPCR for synectin expression. qPCR revealed a statistically significant increase in synectin mRNA levels compared with control samples, $n=4$. Tissue was obtained from patients with liver cirrhosis or matched controls. mRNA and cell lysates were isolated from these samples and analyzed by qPCR (C) or Western blot (D, samples were run on the same gel but were noncontiguous). Both Synectin and $\alpha$-SMA were increased at the mRNA and protein level in cirrhotic patients compared with controls. (Control, $n=5$; cirrhosis, $n=6$ for qPCR. Control, $n=8$; cirrhosis, $n=5$ for Western blot.) (E) Proposed mechanism of synectin regulation of the PDGFR- $\alpha$ and $-\beta$ isoforms is shown in the illustration. Data are expressed as mean \pm SEM. Student's unpaired $t$ test was used to analyze the differences between groups for statistical significance $\left({ }^{*} P<0.05,{ }^{* *} P<0.001,{ }^{* *} P<0.0001\right)$.

with PDGF-bb being the most robust since it activates both PDGFR homodimers and heterodimers. Interestingly, in the presence of PDGF-aa, which is specific for the PDGFR- $\alpha$ homodimer, there was no significant increase in HSC migration in the synectin-knockdown cells, but there was still migration noted in the control cells (Figure 7D). In contrast, while there was a migratory response in synectin-knockdown HSC in response to PDGF-dd, the ligand that activates the PDGFR- $\beta$ homodimer, this response was significantly less than WT HSC. This data highlights differences in the downstream functional response to the PDGFR isoforms and further emphasizes the importance of synectin regulation of both PDGFR isoforms.

Synectin is upregulated in a murine pulmonary fibrosis and human cirrhosis. Given the importance of synectin in liver fibrosis, we next determined if synectin might be important in other fibrotic processes. We obtained isolated mouse lung fibroblasts from a bleomycin model of pulmonary fibrosis (15). Similar to our data in HSC, we found significant increases in synectin, PDGFR- $\beta$, and SMA in the fibroblasts isolated from the bleomycin-exposed mice suggesting that the effects we observed in liver may be generalized to fibrogenesis in other organs (Figure 8A). A similar effect was noted in human lung fibroblasts isolated from patients with idiopathic pulmonary fibrosis (Figure 8B). Finally, as synectin was increased in mouse models of liver 
Table 1. Biospecimen annotation

\begin{tabular}{|c|c|c|c|}
\hline & Controls & Cirrhotics & $P$ value \\
\hline Age (years) & $44 \pm 3.5$ & $52 \pm 6.0$ & 0.29 \\
\hline Sex (\% Female) & $100 \%$ & $66 \%$ & \\
\hline
\end{tabular}

fibrosis and lung fibrosis, we next sought to determine if synectin was also increased in human cirrhotic livers. We found significant increases of synectin by qPCR and Western blotting in liver samples from patients with cirrhosis when compared with noncirrhotic controls (Figure 8, C and D, and Table 1). Taken together, these results show that synectin is increased in human cirrhotic livers, making synectin an interesting target for future drug development.

\section{Discussion}

Synectin has been described as a key protein in arteriogenesis, cancer development and metastatic progression, and neuronal function, but the role of synectin in liver pathology has not been previously investigated. Consequently, our experimental design involved observations in patient-derived liver tissue, the use of genetically engineered mice and cells, the generation of genome-wide expression profiles, examination of chromatin dynamic events that regulate the expression of the fibrogenic PDGFR- $\beta$, the autophagic degradation of the fibrogenic PDGFR- $\alpha$, and the downstream functional consequences of synectin loss in HSC. Notably, the results of these experiments led us to define, for the first time to our knowledge, that synectin plays a fundamental role in the regulation of PDGF signaling, the regulation of PDGF receptors, and the expression of fibrogenic gene expression networks. In total, these findings increase our understanding of molecular mechanisms that participate in the development of liver fibrosis by outlining a membrane-to-nucleus pathway that is critical for this phenomenon (Figure $8 \mathrm{E}$ ). Thus, we discuss our findings in greater detail in the context of their potential novelty and potential biomedical significance.

We were able to find significant differences in the mRNA expression of key genes in the fibrotic pathway by use of the unbiased method of mRNA-Seq including PDGFR- $\beta$. qPCR of PDGFR- $\beta$ confirmed the mRNA-Seq data. Interestingly, the levels of the PDGFR- $\alpha$ mRNA were not altered by synectin knockdown, highlighting that the 2 PDGFR isoforms were regulated by different mechanisms in synectin-knockdown HSC. The role of epigenetic changes in the development of liver disease has been gaining considerable interest, as there is increasing evidence of epigenetic modifications in the development of cirrhosis (16). Given the global changes noted in synectin-knockdown HSC, we examined histone protein modifications that alter binding affinity to open or close specific genome loci for transcription initiation and elongation. To examine this further, we pursued ChIP, targeting specific histone modifications within the PDGFR- $\beta$ gene locus guided by ENCODE database (www.encodeproject.org). In exploring PDGFR- $\beta$ regulation, we were able to confirm key epigenetic changes at the PDGFR- $\beta$ gene locus. Interestingly, we find that epigenetic markers H3K27ac is actively involved in PDGFR- $\beta$ transcription. As p300 is known to acetylate $\mathrm{H} 3$ at K27, we examined the role of synectin knockdown on p300. While p300 is classically regulated by phosphorylation $(17,18)$, we did not observe changes in its phosphorylation status upon synectin knockdown. However, we noted a striking difference in the cellular location of $\mathrm{p} 300$, which may represent an important mechanism of regulating p300 activity by preventing its nuclear localization and, therefore, its histone acetylation activity. Such p300 nuclear translocation could occur potentially through synectin binding to the myosin VI motor complex, which allows for both antegrade and retrograde vesicle trafficking (8), but further work is needed to clarify the mechanism of synectin on p300 cellular localization.

In addition to potential advances that this study makes regarding how synectin epigenetically regulates PDGFR- $\beta$, we show a distinctly different mechanism of synectin regulation of PDGFR- $\alpha$ through autophagic degradation. Indeed, prior work has highlighted differential regulation of the PDGFR isoforms as HSC undergo activation and the emerging importance of PDGFR- $\alpha$ in HSC activation and liver fibrosis (5, 19-21). Autophagy flux has been shown to be decreased in multiple hepatic cell types during liver injury and disease 
(22-24), including nonalcoholic fatty liver disease (25), chronic hepatitis C infection (26), and acetaminophen toxicity (27). However, autophagy has also been shown to increase in HSC during activation $(22,28,29)$. It is hypothesized that the increased autophagy within HSC is necessary for lipid breakdown to provide the necessary energy source to undergo activation through the process of lipophagy (30). Such dichotomous findings are noted in cancer literature, where autophagy has been reported as both promotional and inhibitory for tumor growth (31-35). These divergent findings likely reflect the complexity of the role of autophagy in vivo. Our data demonstrate that synectin can selectively regulate specific protein targets to maintain their active conformation or, alternatively, their autophagic degradation with PDGFR- $\alpha$ as a prototype in this study. It is possible that autophagy may have distinct effects on receptor degradation (what some have referred to as precision autophagy; ref. 36) and lipid breakdown (lipophagy), with cell phenotype reflected by the varying effects of both processes. It is also possible that, in the scenario of HSC activation and increased HSC autophagy, the upregulation of synectin may protect PDGFR- $\alpha$ from degradation. Increasing evidence suggests that RTKs and other signaling proteins can also be regulated and degraded through selective autophagy, including EGFR (37), FLT3 (38), GLUT1 (39), and Src (40). Synectin shares the same myosin VI binding site as target of Myb1 membrane trafficking protein 1 (TOM1), a known autophagy mediator, thus supporting the premise that synectin can regulate the autophagic fate of specific proteins (41). Despite this premise, there is limited data demonstrating this level of action of a scaffold protein such as synectin.

Pathway analysis of our RNA-Seq data revealed that synectin regulates a series of targets within the hepatic fibrosis pathway above and beyond PDGFR. Furthermore, analysis of cellular function showed a general downregulation of genes involved in cellular migration and proliferation. Our in vitro studies in HSC confirmed that both migration and proliferation, 2 hallmark phenotypes of HSC activation, were inhibited in synectin depleted HSC. Additionally, in vivo studies in mice with selective knockdown of synectin from HSC showed significant reduction in fibrosis supporting its specific effect in vivo. Finally, analysis of human cirrhotic livers demonstrated elevated synectin at both the mRNA and protein levels. These results provide evidence that understanding the role of synectin in the development of fibrosis warrants further mechanistic and therapeutic investigation. Although synectin is a known binding partner of NRP-1 (9), the effects of synectin on fibrosis may be broader, given that synectin — unlike NRP-1 — regulates both PDGFR isoforms. While potential human therapies targeting both molecules are evolving, NRP-1 targeting has focused on a neutralizing antibody that can target the extracellular domain of the protein (6), while synectin targeting has focused on small molecules that can be internalized by target cells and act as a neutralizing peptide (42). Given the expression of synectin in multiple liver cell types, it follows that synectin may regulate diverse functions in liver beyond fibrosis; therapies may require HSC selectivity, and caution would be needed for possible off-target effects.

In conclusion, synectin promotes PDGF-dependent HSC activation by diverting PDGFR- $\alpha$ from the selective autophagy pathway and epigenetic regulation of PDGFR- $\beta$. Given the significant role of PDGFR in the development of cirrhosis, this makes synectin a potentially novel target at a therapeutic-development level. At a mechanistic level, these results expand our understanding of how scaffolding proteins can mediate the fate of tyrosine kinase receptors not only through more classic alteration in trafficking, in this case to the precision autophagy pathway, but also through epigenetic changes, thereby stimulating directions for robust future investigation.

\section{Methods}

Cell lines and transfection. Human primary HSCs (ScienCell Research Laboratories) and LX-2 were cultured in DMEM (Thermo Fisher Scientific) supplemented with 10\% FBS (Atlanta Biologicals Inc.) and penicillin/streptomycin (Thermo Fisher Scientific). Small interfering RNA (siRNA) for NRP-1 and ATG5 (catalogs SI00066787 and SI02663206) were purchased from Qiagen. Synectin short hairpin RNAs (shRNA) were purchased from MilliporeSigma (accession numbers NM_005716.2-1083s1c1 and NM_005716.2 1045s1c1). HSC lentiviral transduction of shRNA was carried out by incubation with viral particles for 48 hours in DMEM with 10\% FBS and polybrene, followed by selection with puromycin for 24 hours.

Murine cells. Murine cells used in these studies were isolated from WT mice, Synectin ${ }^{\mathrm{f} / \mathrm{fl}}$ mice and Col ${ }^{\mathrm{cre}} /$ Synectin ${ }^{\mathrm{f} / \mathrm{fl}}$ mice. Hepatocytes, liver endothelial cells, and HSCs were isolated from mice as described (43). Experiments were performed from pooled cells from 3-4 mice. Isolated HSC were transduced in vitro with adenovirus Ad-Cre-eGFP, as we described previously (6). Ad-LacZ was used as a control. Lysates from the cells were collected for Western blot to confirm the loss of synectin and PDGFR isoforms. 
Compounds and ligands. MG132, bafilomycin, and 3-MA were purchased from MilliporeSigma and used at concentrations of $25 \mu \mathrm{M}, 10 \mu \mathrm{M}$, and $1 \mu \mathrm{M}$, respectively, in basal media overnight unless otherwise stated in the figure legends. When compounds were used in knockdown cells, the knockdown was performed prior to incubation with the compound. PDGF refers to PDGF-bb (R\&D Systems; $10 \mathrm{ng} / \mathrm{ml}$ ) unless otherwise stated in the text. HSCs were serum starved overnight. HSCs transduced with control shRNA or synectin shRNA were treated with PDGF-bb $(10 \mathrm{ng} / \mathrm{ml})$ for 2 hours or longer and were harvested for RNA isolation, mRNA-Seq experiments, or other experiments.

Antibodies. Primary antibodies were used at concentration of 1:1,000 (except for GAPDH and $\beta$-actin, which were $1: 5,000$ ) in $5 \%$ skim milk/Tris-buffered saline/ $1 \%$ Tween 20 with overnight incubation at $4^{\circ} \mathrm{C}$. Secondary antibodies (donkey anti-rabbit + HRP or sheep anti-mouse + HRP) were used at a concentration of 1:1,000 (except for GAPDH and $\beta$-actin, which were 1:3,000) for 1 hour at room temperature in Tris-buffered saline/1\% Tween 20. Membranes were developed with luminol solution. Primary antibodies for PDGFR- $\alpha$ (catalog 3164), PDGFR- $\beta$ (catalog 3169), p62 (catalogs 5114 and 7695), pPDGFR- $\beta$ (catalog 3170), ATG5 (catalog 12994), AKT (catalog 9272), and pAKT (catalog 4060) were purchased from Cell Signaling Technology. GAPDH was from MilliporeSigma (catalog AB2302). Synectin antibody was from Proteus Bioscience (catalog 25-6792). $\beta$-Actin antibody was from MilliporeSigma (catalog MABT523).

Plasmid and adenovirus construction. Full-length PDGFR- $\alpha$ (plasmid 23892) and PDGFR- $\beta$ (plasmid 23893) plasmids were purchased from Addgene. cDNA was first subcloned into the TA vector as an intermediate step. Not1/Xho1 (New England Biolabs) restriction enzymes were used to digest PDGFR- $\alpha$, and Not1/Sal1 (New England Biolabs) were used to digest PDGFR- $\beta$ prior to ligation into the AdEASY-FLAG shuttle vector. The AdEASY adenovirus generation was performed as described previously (9).

$R N A$ isolation, $c D N A$ synthesis, and $q P C R$. Total RNA was extracted from cells, mouse tissue, and human tissue according to the manufacturer's instructions using an RNeasy kit (Qiagen), and $5 \mu \mathrm{g}$ was used for cDNA synthesis with oligo (dT) primer using SuperScript III first strand synthesis system for reverse transcription PCR (RT-PCR) (Invitrogen) per the manufacturer's protocol. Real-time PCR was performed in a total 25- $\mu$ l volume reaction using Sybr Green Master Mix and the 7500 real-time PCR system (both from Applied Biosystems), according to the manufacturer's instructions. RT-PCR analysis was performed with the following primer sets: human synectin forward, 5' - GCTGGAGAGTTACATGGGTATC - 3'; human synectin reverse, 5' - TCAGGGAAGGCAAAGTCAC - 3'; mouse synectin forward, 5' - GAAGGTGGATGACTTGCTAGAG - 3'; and mouse synectin reverse, 5' - TCATCTGGGAATGCGAAGTC - 3'; human PDGFR- $\alpha$ forward, 5' - GCGGCCGCATGGGGACTTCCCATCC - 3'; human PDGFR- $\alpha$ reverse, 5' - CTCGAGCAGGAAGCTGTCTTCCACC - 3'; human PDGFR- $\beta$ forward, 5' - AGTGATGTCTGGTCTTTTGGG - 3'; and human PDGFR- $\beta$ reverse, 5' - TGGCATTGTAGAACTGGTCG - 3'. Amplification of human GAPDH and mouse $\beta$-actin was performed in the same reaction for respective samples as internal controls. Each experiment was done in triplicate.

RNA-Seq and analysis. mRNA-Seq libraries were prepared and were sequenced on an Illumina HiSeq 2000 instrument in the Mayo Clinic Center for Individualized Medicine Medical Genomics Facility. Sequence reads from RNA-Seq samples were aligned to the human genome hg19 and gene annotations from Refseq gene using TopHat v2.05. Cufflinks v2.0.2 was used to calculate reads per kilobase of transcript per million mapped reads (RPKM) values of genes. Differential gene expression was analyzed by Cuffdiff using cutoff FDR $<0.05$ and $P<0.05$. Other details are as described previously (44). RNA-Seq data has been deposited in the Gene Expression Omnibus (GEO), accession number GSE93629.

Phospho-RTK assay. HSCs were serum starved overnight. HSCs transduced with control shRNA or synectin shRNA were treated with PDGF-bb $(10 \mathrm{ng} / \mathrm{ml})$ or vehicle $(0.1 \% \mathrm{BSA}$ in $4 \mathrm{mM} \mathrm{HCl})$ for 10 minutes and then harvested for assessment of phosphorylation status of receptor tyrosine kinases. For this purpose, a commercially available human Phospho-Receptor Tyrosine Kinase Array Kit ( catalog ARY001B, R\&D Systems) was used according to the manufacturer's protocol.

ChIP analysis. ChIP was performed with EZ-Magna ChIP (MilliporeSigma, catalog 17-408) with specific immunoprecipitating antibodies such as $\mathrm{H} 3 \mathrm{~K} 4 \mathrm{Me} 3, \mathrm{H} 3 \mathrm{~K} 27 \mathrm{Me} 3$, or H3K27ac with the negative control, normal rabbit IgG as previously described (45).

Protein sequence analysis and site-directed mutagenesis. The Homo sapien amino acid sequence for both PDGFR- $\alpha$ (Universal Protein Knowledgebase [UniProtKB/Swiss-Prot] identification number P16234.1) and PDGFR- $\beta$ (UniProtKB/Swiss-Prot identification number P09619.1) were downloaded in FASTA format from the National Center for Biotechnology Information protein database (http://www.ncbi.nlm.nih.gov/protein). 
The Prosite (http://prosite.expasy.org/) database was used to identify known ubiquitin sites. Known ubiquitin sites were examined on the sequence alignment of PDGFR- $\alpha$ and PDGFR- $\beta$ performed by CLUSTAL 2.1 (http://www.ebi.ac.uk/services/proteins). PDGFR- $\alpha$ amino acid sequences were obtained from 7 species (Xenopus laevis, Gallus gallus, Homo sapiens, Mus musculus, Rattus norvegicus, Fugu rubripes, and Danio rerio) and aligned using CLUSTAL 2.1. Weblogo (http://weblogo.berkeley.edu/logo.cgi) was used to examine domain conservation around known ubiquitin sites. Site-directed mutagenesis was performed as directed in Results.

MS analysis. The SDS-PAGE gel bands of the HSC samples (with or without PDGF treatment) were prepared for MS analysis as per Mayo Proteomics core facility and went through the following procedures. First, silver stained gel bands are destained, and proteins were digested in situ, followed by peptide extraction. Then, the pooled extracts were concentrated, and the proteins were identified by nano-flow liquid chromatography electrospray tandem MS (nanoLC-ESI-MS/MS). Tandem mass spectra were extracted, and all MS/MS samples were analyzed searching the Swiss-Prot March 2014 human protein database (40,620 entries), including a decoy reverse database and assuming the digestion enzyme trypsin. GlyGly of lysine were specified in Mascot as variable modifications (Matrix Science). Peptide identifications were accepted if they could be established at greater than $95 \%$ probability and contained at least 2 identified peptides $(46,47)$.

Cellular imaging. HSC were plated on glass chamber slides coated with collagen I (MilliporeSigma, 10 $\mathrm{ng} / \mathrm{ml}$ ), fixed with $4 \%$ paraformaldehyde (MilliporeSigma) for 5 minutes, permeabilized with $0.1 \%$ Triton-X (Thermo Fisher Scientific) for 2 minutes, and blocked for 1 hour with 10\% FBS. Primary antibodies were incubated overnight at $4^{\circ} \mathrm{C}$ at concentrations recommended by the manufacture. Secondary antibodies conjugated with Alexa fluorophores were purchased from Molecular Probes and incubated for 1 hour at room temperature. Images were obtained at $63 \times$ with Zeiss LSM 5 Pascal, and images were processed with LSM image software. Images were analyzed with ImageJ $(\mathrm{NIH})(48)$. For the animal studies, ImageJ was used to quantify the average signal intensity of the green channel from 3 separate fields per mouse. For colocalization, the JACoP plug-in was used to quantify the Pearson's coefficient (49). Images used for quantification were from 3 separate experiments with 2 fields per experiment analyzed.

$I P$. Primary antibody ( $5 \mu \mathrm{g}$ ) was bound to protein-G beads (GE Healthcare) by incubation in $4^{\circ} \mathrm{C}$ for 2 hours prior to IP. Cell lysate $(150 \mu \mathrm{g})$ was incubated overnight with protein-G/primary antibody beads. After 3 washes with phosphate buffered saline, the beads were boiled with protein loading buffer with DTT. The loading buffer without beads was run on a polyacrylamide gel and transferred onto a nitrocellulose membrane as described previously (6).

Functional assays. LX2 cells were grown to a confluent monolayer in a 6-well tissue culture plate prior to scratch being induced with a $10-\mu 1$ pipette tip. Media was changed with appropriate stimulant at time of scratch. Cells were imaged at 0 and 6 hours after scratch. The total area of the scratch was measured using ImageJ software, and the percent migration area was calculated by the following formula (area time $0 \mathrm{~h}$ - area time $6 \mathrm{~h}$ )/area time $0 \mathrm{~h}$. The Boyden chamber, MTS, and BrdU assays were carried out as previously described $(6,50,51)$. TAT RGS-GAIP peptide to block the PDZ domain of synectin was synthesized based on prior publication (14). Sequence of blocking peptide was YGRKKRRQRRR ppq LQGPSQSSEA and the sequence of scramble peptide was YGRKKRRQRRR ppq LQGPSQSSEA. Two-chamber valve-enabled microfluidic devices were designed and fabricated as previously described (52). Two cell chambers $(3.5 \times 7.5 \times 0.1 \mathrm{~mm}$ [length $\times$ width $\times$ height] $)$ were separated by a barrier with an array of microgrooves $(100 \times 30 \times 10 \mu \mathrm{m})$. On the top of the 2 chambers, there was a pressure chamber to manipulate the opening status of these microgrooves. The microfluidic platforms were first sterilized and then coated with collagen solution $(0.2 \mathrm{mg} / \mathrm{ml})$ for 4 hours at $37^{\circ} \mathrm{C}$ inside a cell incubator. The devices were washed with $1 \times$ PBS and then filled with culture media. Before seeding with cells, the valve was activated by injecting deionized water into the pressure chamber. The microgrooves were sealed to isolate the 2 cell chambers. After removing the excess media, a $15-20 \mu 1$ of the cell suspension at a density of $\sim 5,000$ cells per $\mu 1$ of media was added to 1 chamber with pillars. The other chamber was used to add chemoattractant PDGF-bb into media. The microfluidic platforms were then placed inside a cell culture incubator with $5 \% \mathrm{CO}_{2}$ at $37^{\circ} \mathrm{C}$ to allow for cell attach. After 1 hour, the seeding solution was removed and approximately $200 \mu 1$ of culture media was added to each reservoir. Cells were treated with scrambled or RGS-GAIP peptide. Before imaging, the deionized water in the pressure chamber was released. The microgrooves were reopened to allow for migration of cells to another chamber because of chemoattractant gradient. Subsequently, the device 
was mounted onto the microscopy stage for imaging. The time-lapse images (16 hours long with a 10-minute interval) were taken using a Lionheart FX automated live cell microscope (BioTek). Cell migration was analyzed by ImageJ software.

RACl activity assay. HSCs were serum starved overnight. HSCs transduced with control shRNA or synectin shRNA were treated with PDGF-bb $(10 \mathrm{ng} / \mathrm{ml})$ or vehicle $(0.1 \% \mathrm{BSA}$ in $4 \mathrm{mM} \mathrm{HCl})$ for 3 minutes and were harvested for Rac1 activity assay using a commercially available Active Rac1 Detection Kit (Cell Signaling Technology, catalog 8815) according to the manufacturer's protocol.

Animal studies. Synectin ${ }^{\mathrm{f} / \mathrm{fl}}$ mice were previously described in Moraes et al. (53). Synectin ${ }^{\mathrm{f} / \mathrm{fl}} \mathrm{mice}$ were crossed with $\mathrm{Col}^{\text {cre }}$ mice (54) to generate $\mathrm{Col}^{\text {cre }} /$ Synectin ${ }^{\mathrm{f} / \mathrm{fl}}$ mice that are genetically deficient of synectin in myofibroblasts. Cre-negative littermates were used for control. Eight- to 10-weeks old Synectin $^{\mathrm{fl} / \mathrm{fl}}$ mice and $\mathrm{Col}^{\text {cre }} /$ Synectin ${ }^{\mathrm{f} / \mathrm{fl}}$ mice with an initial body weight of approximately $25 \mathrm{~g}$ underwent twice-weekly i.p. injections of $0.5 \mathrm{mg} / \mathrm{kg}$ body weight of $\mathrm{CCl}_{4}$ for 6 weeks. Livers were harvested at week 6. Tissue was taken for Western blotting, hydroxyproline assay, qPCR, and immunofluorescence as previously described $(55,56)$. Similarly, we used another model of liver fibrosis in mice. Eight-weekold $\mathrm{Col}^{\mathrm{cre}} /$ Synectin ${ }^{\mathrm{f} / \mathrm{fl}}$ and Synectin ${ }^{\mathrm{f} / \mathrm{fl}}$ were divided into 2 groups. One group underwent BDL surgery as described previously (56), and the other group received sham (control) surgery. Mice were sacrificed 4 weeks after the surgeries, and liver tissue was analyzed for liver fibrosis markers. cDNA from isolated lung fibroblast that had been exposed to inhaled bleomycin or vehicle for 14-21 days were a gift from Daniel J. Tschumperlin (Mayo Clinic) (15).

Human samples. RNA was extracted from liver biopsies of control and alcoholic cirrhotic patients as described previously (57). Patient demographics are shown in Table 1. Protein was extracted using RIPA buffer at $100 \mu 1$ to $1 \mathrm{mg}$ of tissue ratio.

Statistics. Experiments were performed at least 3 independent times and numerical data expressed as mean \pm SEM. Student's unpaired $t$ test was used to analyze the differences between 2 groups. Oneway ANOVA with Bonferroni's multiple comparison test was used to analyze multiple groups for statistical significance. A $P$ value less than 0.05 was considered significant.

Study approval. All animal experiments were approved by IACUC and carried out in accordance with institutional guidelines (Mayo Clinic). Patient sample use was approved by the IRB at the Mayo Clinic.

\section{Author contributions}

MCD contributed study design, synectin biochemical experiments, PDGFR- $\beta$ mutant biochemical experiments, imaging with LC3B-GFP construct, human sample studies, scratch assay, bioinformatics analysis, quantification of confocal imaging, human sample analysis, figure preparation, and manuscript writing/editing. UY contributed NRP-1/synectin biochemical experiments, cell culture and transfections, Boyden chamber assays, mouse models, primary cell isolations, immunostaining and quantification of confocal imaging, figure preparation, RNA-Seq, and manuscript preparation/editing. HY contributed adenoviral preparation, confocal microscopy with adenoviral constructions, and the $\mathrm{CCl}_{4}$ mouse model. FL contributed histone marker, p300 and ChIP experiments, and mouse sample analysis. VKV contributed cloning of the PDGFR- $\alpha$ and PDGFR- $\beta$ (WT and mutant) constructs. EK contributed overexpressed PDGFR K971A mutant and worked with BDL mouse model. JM contributed PDGFR- $\beta$ mutant biochemical experiments and hydroxyproline assay. TG worked with BrdU assay, Rac activity, and phosphor-RTK assay. JPA worked with BDL mouse model. SC contributed study design, NRP-1 biochemical experiments, co-IP assay, mRNA-Seq bioinformatics analysis, mentoring, and manuscript writing/editing. VHS contributed study design, manuscript writing, manuscript editing, and mentoring. TMDA did mice BDL surgeries. MS provided Synectin ${ }^{\mathrm{f} / \mathrm{fl}}$ mice and assistance regarding experiments with those mice. DM provided us with NRP-1 mutant constructs and advice regarding study design. TK provided $\mathrm{Co}^{\text {cre }}$ mice and assisted with experiments using those mice. DAB assisted with experiments with $\mathrm{Col}^{\text {cre }}$ mice studies. RU assisted with designing and analyzing RNA-Seq experiments. G. Ligresti assisted with experiments with mouse model of bleomycin-induced lung fibrosis. YG provided us with microfluidic chambers and assisted in experiments utilizing those chambers. G. Lomberk assisted with designing RNA-Seq experiments. DJT assisted with experiments relating to pulmonary fibrosis in both human patient samples and mouse models of bleomycin-induced lung fibrosis. AR provided us with microfluidic chambers and assisted in experiments with those chambers. All authors reviewed the results and approved the final version of the manuscript. 


\section{Acknowledgments}

This work was supported in part by the NIH R01 DK59615 and R01 AA21171. HY is supported by National Natural Science Foundation of China (31400761) and Beijing Natural Science Foundation (7142079). FL is supported by National Natural Science Foundation of China (81472458) and Fujian Science and Technology Program (2017R1036-3). TG is supported by the Swiss National Science Foundation (P2ZHP3_168561).

Address correspondence to: Vijay H. Shah or Sheng Cao, Mayo Clinic, $2001^{\text {st }}$ Street SW, Rochester, Minnesota 55905, USA. Phone: 507.255.6028; Email: shah.vijay@mayo.edu (V.H. Shah); cao.sheng@mayo.edu (S. Cao).

1. Henderson NC, Iredale JP. Standing down the guard: stellate cells leave quietly. Gastroenterology. 2012;143(4):890-892.

2. Granzow M, et al. Angiotensin-II type 1 receptor-mediated Janus kinase 2 activation induces liver fibrosis. Hepatology. 2014;60(1):334-348.

3. Mogler C, et al. Hepatic stellate cell-expressed endosialin balances fibrogenesis and hepatocyte proliferation during liver damage. EMBO Mol Med. 2015;7(3):332-338.

4. Iwakiri Y. Pathophysiology of portal hypertension. Clin Liver Dis. 2014;18(2):281-291.

5. Breitkopf K, Roeyen Cv, Sawitza I, Wickert L, Floege J, Gressner AM. Expression patterns of PDGF-A, -B, -C and -D and the PDGF-receptors alpha and beta in activated rat hepatic stellate cells (HSC). Cytokine. 2005;31(5):349-357.

6. Cao S, et al. Neuropilin-1 promotes cirrhosis of the rodent and human liver by enhancing PDGF/TGF-beta signaling in hepatic stellate cells. J Clin Invest. 2010;120(7):2379-2394.

7. Reichenbach V, et al. Adenoviral dominant-negative soluble PDGFR $\beta$ improves hepatic collagen, systemic hemodynamics, and portal pressure in fibrotic rats. J Hepatol. 2012;57(5):967-973.

8. Katoh M. Functional proteomics, human genetics and cancer biology of GIPC family members. Exp Mol Med. 2013;45:e26.

9. Yaqoob U, et al. Neuropilin-1 stimulates tumor growth by increasing fibronectin fibril assembly in the tumor microenvironment. Cancer Res. 2012;72(16):4047-4059.

10. Chittenden TW, et al. Selective regulation of arterial branching morphogenesis by synectin. Dev Cell. 2006;10(6):783-795.

11. Metsalu T, Vilo J. ClustVis: a web tool for visualizing clustering of multivariate data using Principal Component Analysis and heatmap. Nucleic Acids Res. 2015;43(W1):W566-W570.

12. Klionsky DJ, et al. Guidelines for the use and interpretation of assays for monitoring autophagy (3rd edition). Autophagy. 2016;12(1):1-222.

13. Sigrist CJ, et al. New and continuing developments at PROSITE. Nucleic Acids Res. 2013;41(Database issue):D344-D347.

14. Wang L, et al. RGS-GAIP-interacting protein controls breast cancer progression. Mol Cancer Res. 2010;8(12):1591-1600.

15. Liu F, et al. Feedback amplification of fibrosis through matrix stiffening and COX-2 suppression. J Cell Biol. 2010;190(4):693-706

16. Lee YA, Wallace MC, Friedman SL. Pathobiology of liver fibrosis: a translational success story. Gut. 2015;64(5):830-841.

17. Chen YJ, Wang YN, Chang WC. ERK2-mediated C-terminal serine phosphorylation of p300 is vital to the regulation of epidermal growth factor-induced keratin 16 gene expression. J Biol Chem. 2007;282(37):27215-27228.

18. Sang N, Stiehl DP, Bohensky J, Leshchinsky I, Srinivas V, Caro J. MAPK signaling up-regulates the activity of hypoxia-inducible factors by its effects on p300. J Biol Chem. 2003;278(16):14013-14019.

19. Hayes BJ, et al. Activation of platelet-derived growth factor receptor alpha contributes to liver fibrosis. PLoS One. 2014;9(3):e92925.

20. Kikuchi A, Monga SP. PDGFR $\alpha$ in liver pathophysiology: emerging roles in development, regeneration, fibrosis, and cancer Gene Expr. 2015;16(3):109-127.

21. Liu C, et al. PDGF receptor- $\alpha$ promotes TGF- $\beta$ signaling in hepatic stellate cells via transcriptional and posttranscriptional regulation of TGF- $\beta$ receptors. Am J Physiol Gastrointest Liver Physiol. 2014;307(7):G749-G759.

22. Hernández-Gea V, et al. Autophagy releases lipid that promotes fibrogenesis by activated hepatic stellate cells in mice and in human tissues. Gastroenterology. 2012;142(4):938-946.

23. Marciniak SJ, Lomas DA. Alpha1-antitrypsin deficiency and autophagy. N Engl J Med. 2010;363(19):1863-1864.

24. Sasaki M, Miyakoshi M, Sato Y, Nakanuma Y. Autophagy may precede cellular senescence of bile ductular cells in ductular reaction in primary biliary cirrhosis. Dig Dis Sci. 2012;57(3):660-666.

25. Liu HY, et al. Hepatic autophagy is suppressed in the presence of insulin resistance and hyperinsulinemia: inhibition of FoxO1-dependent expression of key autophagy genes by insulin. J Biol Chem. 2009;284(45):31484-31492.

26. Rautou PE, et al. Changes in autophagic response in patients with chronic hepatitis C virus infection. Am J Pathol. 2011;178(6):2708-2715.

27. Ni HM, et al. Liver-specific loss of Atg5 causes persistent activation of Nrf2 and protects against acetaminophen-induced liver injury. Toxicol Sci. 2012;127(2):438-450.

28. Thoen LF, et al. A role for autophagy during hepatic stellate cell activation. J Hepatol. 2011;55(6):1353-1360.

29. Thoen LF, Guimarães EL, Grunsven LA. Autophagy: a new player in hepatic stellate cell activation. Autophagy. 2012;8(1):126-128.

30. Czaja MJ, et al. Functions of autophagy in normal and diseased liver. Autophagy. 2013;9(8):1131-1158.

31. Jiang X, Overholtzer M, Thompson CB. Autophagy in cellular metabolism and cancer. J Clin Invest. 2015;125(1):47-54.

32. Ojha R, Bhattacharyya S, Singh SK. Autophagy in Cancer Stem Cells: A Potential Link Between Chemoresistance, Recurrence, and Metastasis. Biores Open Access. 2015;4(1):97-108.

33. Rausch V, et al. Autophagy mediates survival of pancreatic tumour-initiating cells in a hypoxic microenvironment. $J$ Pathol. 2012;227(3):325-335.

34. Song YJ, et al. Autophagy contributes to the survival of CD133+ liver cancer stem cells in the hypoxic and nutrient-deprived tumor microenvironment. Cancer Lett. 2013;339(1):70-81. 
35. White E. The role for autophagy in cancer. J Clin Invest. 2015;125(1):42-46.

36. Kimura T, et al. TRIM-mediated precision autophagy targets cytoplasmic regulators of innate immunity. J Cell Biol. 2015;210(6):973-989.

37. Jones S, Cunningham DL, Rappoport JZ, Heath JK. The non-receptor tyrosine kinase Ack1 regulates the fate of activated EGFR by inducing trafficking to the p62/NBR1 pre-autophagosome. J Cell Sci. 2014;127(Pt 5):994-1006.

38. Larrue C, et al. Proteasome inhibitors induce FLT3-ITD degradation through autophagy in AML cells. Blood. 2016;127(7):882-892.

39. Bhattacharya S, et al. GAIP interacting protein C-terminus regulates autophagy and exosome biogenesis of pancreatic cancer through metabolic pathways. PLoS One. 2014;9(12):e114409.

40. Sandilands E, Schoenherr C, Frame MC. p70S6K is regulated by focal adhesion kinase and is required for Src-selective autophagy. Cell Signal. 2015;27(9):1816-1823.

41. Tumbarello DA, Kendrick-Jones J, Buss F. Myosin VI and its cargo adaptors - linking endocytosis and autophagy. J Cell Sci. 2013;126(Pt 12):2561-2570.

42. Patra CR, et al. Chemically-Modified Peptides Targeting the PDZ Domain of GIPC as a Therapeutic Approach for Cancer [published online ahead of print March 10, 2015]. ACS Chem Biol. https://doi.oeg/10.1021/cb500865a

43. Wang R, et al. Hepatic Stellate Cell Selective Disruption of Dynamin-2 GTPase Increases Murine Fibrogenesis through Up-Regulation of Sphingosine-1 Phosphate-Induced Cell Migration. Am J Pathol. 2017;187(1):134-145

44. De Assuncao TM, et al. Development and characterization of human-induced pluripotent stem cell-derived cholangiocytes. Lab Invest. 2015;95(10):1218.

45. de Assuncao TM, et al. New role for Kruppel-like factor 14 as a transcriptional activator involved in the generation of signaling lipids. J Biol Chem. 2014;289(22):15798-15809.

46. Keller A, Nesvizhskii AI, Kolker E, Aebersold R. Empirical statistical model to estimate the accuracy of peptide identifications made by MS/MS and database search. Anal Chem. 2002;74(20):5383-5392.

47. Nesvizhskii AI, Keller A, Kolker E, Aebersold R. A statistical model for identifying proteins by tandem mass spectrometry. Anal Chem. 2003;75(17):4646-4658

48. Schneider CA, Rasband WS, Eliceiri KW. NIH Image to ImageJ: 25 years of image analysis. Nat Methods. 2012;9(7):671-675.

49. Bolte S, Cordelières FP. A guided tour into subcellular colocalization analysis in light microscopy. J Microsc. 2006;224(Pt 3):213-232

50. Das A, et al. Disruption of an SP2/KLF6 repression complex by SHP is required for farnesoid X receptor-induced endothelial cell migration. J Biol Chem. 2006;281(51):39105-39113.

51. Beijer Å, Rosenberger A, Bölck B, Suhr F, Rittweger J, Bloch W. Whole-body vibrations do not elevate the angiogenic stimulus when applied during resistance exercise. PLoS One. 2013;8(11):e80143

52. Gao Y, et al. A versatile valve-enabled microfluidic cell co-culture platform and demonstration of its applications to neurobiology and cancer biology. Biomed Microdevices. 2011;13(3):539-548.

53. Moraes F, et al. Endothelial cell-dependent regulation of arteriogenesis. Circ Res. 2013;113(9):1076-1086.

54. Kisseleva T, et al. Myofibroblasts revert to an inactive phenotype during regression of liver fibrosis. Proc Natl Acad Sci USA 2012;109(24):9448-9453.

55. Cao Y, et al. Neuropilin-1 mediates divergent R-Smad signaling and the myofibroblast phenotype. J Biol Chem. 2010;285(41):31840-31848.

56. Yang L, et al. Vascular endothelial growth factor promotes fibrosis resolution and repair in mice. Gastroenterology. 2014;146(5):1339-50.e1.

57. Lee JS, et al. A novel prognostic subtype of human hepatocellular carcinoma derived from hepatic progenitor cells. Nat Med. 2006;12(4):410-416. 